\title{
AND YET IT MOVES: THE DANGERS OF ARTIFICIALLY FIXING THE MILKY WAY CENTER OF MASS IN THE PRESENCE OF A MASSIVE LARGE MAGELLANIC CLOUD
}

\author{
Facundo A. Gómez ${ }^{1,2,3}$, Gurtina Besla ${ }^{4}$, Daniel D. Carpintero ${ }^{5}$, Álvaro Villalobos ${ }^{6}$, \\ Brian W. O'Shea ${ }^{1,2,7,8}$, and ERIC F. Bell ${ }^{9}$ \\ ${ }^{1}$ Department of Physics and Astronomy, Michigan State University, East Lansing, MI 48824, USA; fgomez@mpa-garching.mpg.de \\ ${ }^{2}$ Institute for Cyber-Enabled Research, Michigan State University, East Lansing, MI 48824, USA \\ ${ }^{3}$ Max-Planck-Institut für Astrophysik, Karl-Schwarzschild-Strasse 1, D-85748, Garching, Germany \\ ${ }^{4}$ Steward Observatory, University of Arizona, 933 North Cherry Avenue, Tucson, AZ 85721, USA \\ ${ }^{5}$ Facultad de Ciencias Astronómicas y Geofísicas, Universidad Nacional de La Plata, Argentina and Instituto de Astrofísica de La Plata, \\ UNLP-Conicet La Plata, Argentina \\ ${ }^{6}$ Astronomical Observatory of Trieste, via G.B. Tiepolo 11, I-34143 Trieste, Italy \\ ${ }^{7}$ Lyman Briggs College, Michigan State University, East Lansing, MI 48825, USA \\ ${ }^{8}$ Joint Institute for Nuclear Astrophysics, USA \\ ${ }^{9}$ Department of Astronomy, University of Michigan, 830 Dennison Building, 500 Church Street, Ann Arbor, MI 48109, USA \\ Received 2014 August 14; accepted 2015 February 9; published 2015 April 2
}

\begin{abstract}
Motivated by recent studies suggesting that the Large Magellanic Cloud (LMC) could be significantly more massive than previously thought, we explore whether the approximation of an inertial Galactocentric reference frame is still valid in the presence of such a massive LMC. We find that previous estimates of the LMC's orbital period and apocentric distance derived assuming a fixed Milky Way (MW) are significantly shortened for models where the MW is allowed to move freely in response to the gravitational pull of the LMC. Holding other parameters fixed, the fraction of models favoring first infall is reduced. Due to this interaction, the MW center of mass within the inner $50 \mathrm{kpc}$ can be significantly displaced in phase-space in a very short period of time that ranges from 0.3 to $0.5 \mathrm{Gyr}$ by as much as $30 \mathrm{kpc}$ and $75 \mathrm{~km} \mathrm{~s}^{-1}$. Furthermore, we show that the gravitational pull of the LMC and response of the MW are likely to significantly affect the orbit and phase space distribution of tidal debris from the Sagittarius dwarf galaxy (Sgr). Such effects are larger than previous estimates based on the torque of the LMC alone. As a result, Sgr deposits debris in regions of the sky that are not aligned with the present-day Sgr orbital plane. In addition, we find that properly accounting for the movement of the MW around its common center of mass with the LMC significantly modifies the angular distance between apocenters and tilts its orbital pole, alleviating tensions between previous models and observations. While these models are preliminary in nature, they highlight the central importance of accounting for the mutual gravitational interaction between the MW and LMC when modeling the kinematics of objects in the MW and Local Group.
\end{abstract}

Key words: galaxies: formation - Galaxy: formation - Galaxy: halo - methods: analytical - methods: numerical methods: statistical

\section{INTRODUCTION}

Recently, a series of studies based on photometric, kinematic, and dynamical arguments have enhanced our current understanding of the orbital history and mass of the Magellanic Clouds system (see, e.g., Kallivayalil et al. 2013, and references therein). The results presented in these studies suggest that the Large Magellanic Cloud (LMC) could be significantly more massive than previously thought. Besla et al. $(2010,2012)$ showed that the observed irregular morphology and internal kinematics of the Magellanic System (in both the gas and stellar components) are naturally explained by interactions between the LMC and the Small Magellanic Cloud (SMC hereafter), rather than gravitational interactions with the Milky Way (MW hereafter). Kallivayalil et al. (2013, hereafter K13) showed that in order for the SMC to be bound to the LMC for periods as large as $2 \mathrm{Gyr}$ (the estimated age of the Magellanic stream) a LMC with a mass greater than $1 \times 10^{11} M_{\odot}$ is required. In addition, based on proper motion measurements obtained using the Hubble Space Telescope $(H S T)$, Besla et al. (2007) and K13 showed that for such massive LMC models, the Magellanic Clouds are likely to be experiencing their first infall toward the MW.
Could the acceleration of the inner regions of the MW induced by such a massive LMC be significant, even if it is experiencing its first pericenter passage? In binary stellar systems, the two stars orbit about a common center of mass that is often exterior to the more massive star. The MW+LMC system may be analogous, where the center of mass of the combined system may be at a non-negligible distance from the Galactic center. A simple back-of-the-envelope calculation suggests that this may indeed be the case. For example, assuming a MW model with a dark matter (DM) halo of viral mass $M_{\mathrm{vir}}=1 \times 10^{12} M_{\odot}$, the mass of the MW enclosed within the LMC present-day position, $R_{\mathrm{LMC}} \approx 50 \mathrm{kpc}$, is approximately $M_{\mathrm{MW}}^{50} \approx 3-4 \times 10^{11} M_{\odot}$. The LMC canonical model adopted by K13, based on the requirement that the LMC and SMC have been a long-lived binary, assumes a total mass of $M_{\mathrm{LMC}}=1.8 \times 10^{11} M_{\odot}$. In this MW+LMC system, the orbital barycenter could be displaced by as much as $\approx 14 \mathrm{kpc}$ from the Galactic center. The associated phase-space displacement of the MW with respect to its orbital barycenter could have a substantial impact on the inferred orbital properties of satellite galaxies, including the LMC itself. In other words, such a massive satellite orbiting the MW at the present day 
could pose a serious challenge to the commonly adopted assumption of an inertial Galactocentric reference frame.

While understanding the motion of the MW and its neighbors is of relevance for many Local Group studies, a deep understanding of the expected response of the MW to the gravitational pull of such a massive LMC is urgently needed for analyses based on orbital integration using present-day phase-space coordinates as initial conditions (ICs). Furthermore, due to the extended nature of the MW stellar halo, not all stars will experience the same acceleration from the LMC. This differential acceleration could introduce observable signatures on the phase-space distribution of extended tidal streams, such as those associated with the Sagittarius dwarf galaxy (Sgr). The Sgr tidal tails span at least $300^{\circ}$ across the sky (Ibata et al. 1997), and have been observed at Galactocentric distances as large as $100 \mathrm{kpc}$ (e.g., Majewski et al. 2003; Newberg et al. 2003; Ruhland et al. 2011; Drake et al. 2013). Indeed, Vera-Ciro \& Helmi (2013) showed that the torque on Sgr exerted by the LMC can introduce non-negligible perturbations to the orbit of Sgr and its distribution of debris. Their work, however, considered an spatially fixed MW model, thus neglecting the dynamical response of the MW to the gravitational pull of the LMC.

The aforementioned perturbations, associated with the plausible presence of a massive LMC, could even influence the determination of the present-day Galactic mass distribution. Multiple observational programs have provided, and will continue to provide, very accurate photometric, astrometric, and spectroscopic information for enormous samples of stars, not only in the Galactic disk but also in the more extended stellar halo (see e.g., York et al. 2000; Perryman et al. 2001; Steinmetz et al. 2006; Keller et al. 2007; Yanny et al. 2009; Barden et al. 2010; Cui et al. 2012; Gilmore et al. 2012). During the last two decades several studies were devoted to the development and application of powerful theoretical and statistical tools that could allow us to efficiently mine these observational data sets. An important goal in many of these studies is to statistically infer the present-day Galactic mass distribution. It is customary for these studies to consider as input data dynamically young and extended stellar streams (e.g., Helmi 2004; Johnston et al. 2005; Law \& Majewski 2010; Koposov et al. 2010; Lux et al. 2012; Sanders \& Binney 2013b; Vera-Ciro \& Helmi 2013; Bonaca et al. 2014; Deg \& Widrow 2014; Gibbons et al. 2014; Price-Whelan et al. 2014). The reason behind this choice is simple: these types of spatially extended streams are expected to approximately delineate the orbit of their corresponding progenitors in phase-space (see Eyre \& Binney 2011; Sanders \& Binney 2013a).

For simplicity, in most of these works the MW's mass distribution has been assumed to be smooth and static, not only structurally but also spatially. Assuming a frozen-mass potential may not strongly affect the results of these analyzes. The MW's mass is not expected to have significantly evolved during the last 2-3 Gyr (e.g., Bullock \& Johnston 2005), a dynamical timescale that pertains to these studies. On the other hand, the assumption that the MW can be regarded as an inertial frame has not been thoroughly tested. If one neglects the presence of the LMC, the MW's accretion activity can be regarded as quiescent during this period of time. However, the degree to which the presence of a massive LMC could significantly affect the statistically inferred parameters that best describe the Galactic potential remains to be studied.

The dangers associated with artificially fixing the MW center of mass have been considered by several authors in the past. One of the first works to explore this was presented by White (1983). Using $N$-body simulations, this study showed that the orbital decay rate of a satellite galaxy is artificially enhanced by fixing the host center of mass. Current analytic prescriptions to model dynamical friction are fine-tuned by calibrating against results of fully self-consistent $\mathrm{N}$-body simulations (e.g., Cora et al. 1997; Benson et al. 2004; Just \& Peñarrubia 2005). More recently, Perryman et al. (2014) discussed the effects that the time evolution of the orientation of the disk angular momentum vector with respect to an initial reference frame could have on Gaia measurements. Such perturbations to the disk angular momentum could be caused by, e.g., the time-dependent accretion of gas (Shen \& Sellwood 2006; Roškar et al. 2010), the predicted tumbling of the Galactic dark matter halo (e.g., Bailin \& Steinmetz 2005; Bryan \& Cress 2007; Vera-Ciro et al. 2011) and by the tidal interaction of a fairly massive LMC (Bekki 2012).

In this work we revisit the problem of a non-inertial MW reference frame by modeling the interaction between the MW, the Sgr, and an LMC that is undergoing its first infall at the present day. We focus our analysis on two possible situations where the response of the MW to the gravitational pull of the LMC could induce significant perturbations: namely, the inferred orbit of the LMC about the MW, and the orbit and tidal debris from the Sgr dwarf galaxy. To this end, we use a variety of different techniques to model the gravitational interaction between these three galaxies. In Section 2 we provide a justification for the LMC mass range explored in our experiments. In Sections 3 and 4 we use smooth analytic representations of the Galactic potentials to characterize the significance of this perturbative effect on the orbital properties of both the LMC and Sgr. In Section 5 we use full $N$-body simulations to explore the consequences of a non-inertial Galactocentric reference frame on the phase-space distribution of the Sgr tidal debris. We conclude and discuss our results in Section 6.

\section{THE MASS OF THE LMC}

In this study we consider LMC mass models that range from $3 \times 10^{10}$ to $2.5 \times 10^{11} M_{\odot}$. We would like to stress that the considered LMC masses are meant to represent the total LMC infall mass up to its virial radius, as opposed to the present-day observational constraint within its optical radius. In this section we justify this mass range and explain why high-mass LMC models are currently favored. We refer the reader to Besla et al. (2014) for a more extended discussion.

Our goal is to explore the effects of a massive LMC on the assumption that the MW can be considered an inertial frame of reference. The mass of the LMC is the dominant uncertainty in the orbital history of the Magellanic Clouds since dynamical friction is proportional to its mass. Moreover, the mass of the LMC also controls the orbit of the SMC, ultimately determining how long the two galaxies have interacted with each other as a binary pair.

Observationally, the total mass of the LMC is only constrained within the optical radius. The LMC has a welldefined rotation curve that peaks at $V_{\text {circ }}=91.7 \pm 18.8 \mathrm{~km} \mathrm{~s}^{-1}$ and remains flat out to at least $8.7 \mathrm{kpc}$ (van der Marel \& 
Kallivayalil 2014). This peak velocity places the LMC squarely on the well-defined baryonic Tully-Fisher relation (McGaugh 2012). This implies a minimum enclosed total mass of $M(8.7 \mathrm{kpc})=1.7 \times 10^{10} M_{\odot}$, and further implies that the LMC is DM-dominated. The total mass of the LMC may be much larger than this, depending on the tidal radius.

There is strong evidence that the stellar disk of the LMC extends to $15 \mathrm{kpc}$ (Majewski et al. 2009; Saha et al. 2010). If the rotation curve stays flat to at least this distance then the total mass enclosed is $M(15 \mathrm{kpc})=V_{\text {circ }}^{2} r / G \sim 3 \times 10^{10} M_{\odot}$. This minimum value is consistent with LMC masses adopted by traditional models of the orbital evolution of the Magellanic Clouds (e.g., Murai \& Fujimoto 1980; Gardiner \& Noguchi 1996). Note however that this estimate only takes into account the total mass of the LMC within $15 \mathrm{kpc}$. Thus, it may significantly underestimate its total infall mass within its virial radius; this is the quantity of interest for this work.

The total dynamical mass of the LMC at infall, up to its virial radius, can be estimated using its baryon fraction. Currently, the LMC has a stellar mass of $2.7 \times 10^{9} M_{\odot}$ and a gas mass of $5.0 \times 10^{8} M_{\odot}$. The baryonic mass of the LMC is thus $M_{\mathrm{bar}}=3.2 \times 10^{9} M_{\odot}$. Using the minimum total mass of $M_{\text {tot }}=3 \times 10^{10} M_{\odot}$, the baryon fraction of the LMC becomes $M_{\mathrm{bar}} / M_{\mathrm{tot}}=11 \%$. This is much higher than the baryon fraction of disks in galaxies like the MW, which is of the order of $3-5 \%$. In the shallower halo potentials of dwarf galaxies, stellar winds should be more efficient, making baryon fractions even lower, not higher.

This analysis is further complicated if material has been removed from the LMC. Fox et al. (2014) have recently estimated the total gas mass (HI and ionized gas) outside the Magellanic Clouds at $2 \times 10^{9}(d / 55 \mathrm{kpc})^{2} M_{\odot}$, with $d$ the distance to the Magellanic stream. If half of this material came from the LMC, as suggested by Nidever et al. (2008), its initial baryon fraction would be $14 \%$-approaching the cosmic value. Note that the bulk of the Magellanic Stream likely resides at distances of order $d=100 \mathrm{kpc}$, rather than $55 \mathrm{kpc}$, in which case the baryon fraction would increase to $\sim 20 \%$.

In order for the baryon fraction to match observational expectations of $f_{\mathrm{bar}} \sim 3-5 \%$, the total mass of the LMC (at least at infall) needs to have been $6-20 \times 10^{10} M_{\odot}$. This higher total mass is consistent with cosmological expectations from halo occupation models that relate a galaxy's observed stellar mass to its halo mass. Using relations from Moster et al. (2013), the mean halo mass for a galaxy with a stellar mass of $2.7 \times 10^{9} M_{\odot}$ is $1.7 \times 10^{11} M_{\odot}$, implying a baryon fraction of $f_{\text {bar }} \sim 2-4 \%$. Because there is a large scatter in halo occupation models, we have considered a maximal halo mass for the LMC of $2.5 \times 10^{11} M_{\odot}$.

The halo occupation model relations are primarily invoked to motivate ICs for a first infall model. As shown in K13 and later in this work, first infall models are obtained in MW-like hosts with a total mass $\approx 1 \times 10^{12} M_{\odot}$, regardless of the total LMC mass (within the range considered here). If the Clouds have only recently been accreted there has not been enough time to severly truncate the LMC halo and, as a result, its current mass should approximately reflect its infall mass; i.e., the mass the LMC halo had upon first crossing the virial radius. Note as well that high-mass LMC models, $>10^{11} M_{\odot}$, are necessary in models of the formation of the Magellanic Stream as they allow for a long-lived ( $4 \mathrm{Gyr})$ LMC/SMC binary configuration. The relative velocity between the Clouds is $\sim 130 \mathrm{~km} \mathrm{~s}^{-1}$; high-mass LMC models (masses of order $10^{11} M_{\odot}$ ) are needed to explain how the LMC can have held on to the SMC if it is moving at such speeds. This argument has been outlined in K13. Based on their parameter space search, and the requirement that the LMC and SMC have been a long-lived binary, we adopt a canonical mass model for the $\mathrm{LMC}=1.8 \times 10^{11} M_{\odot}$.

A very important uncertainty in the kind of analytic orbital integration schemes we employ in this study is the mass evolution of the LMC over time. The arguments laid out in this section are for the required infall mass of the LMC. The subsequent mass loss incurred by the LMC as it orbits about the MW will necessarily cause significant modifications in the orbits presented in the following section. A proper analysis accounting for this effect requires detailed $N$-body simulations that, in principle, are beyond the scope of the simple backward integration scheme presented here. Nonetheless, to validate our assumptions, we will compare the results from one of the MW +LMC mass combinations with those obtained with the corresponding fully self-consistent $N$-body model.

\section{THE ORBIT OF THE LMC ABOUT THE MW}

Our goal in this section is to explore whether artificially fixing the MW center of mass could have significant implications on the inferred orbital properties of the LMC. For this purpose, we will integrate the orbits of different LMC models backwards in time in MW-like hosts that are kept artificially fixed in space and that are also allowed to react to the gravitational pull of the LMC. In order to make a direct comparison with the results presented in K13 we will start by considering smooth, analytic representations of the Galactic potentials. We will later compare our results with those obtained from full $N$-body simulations. The models and methodology are described in Section 3.1. Our results are presented on Section 3.2.

\subsection{Analytic Models \\ 3.1.1. Methodology}

To follow the evolution of the gravitational interaction between the MW and the LMC we used a symplectic leapfrog integration scheme (Springel et al. 2001). Both the host and the satellite are represented with analytic potentials; the center of each one follows the orbit that results from the acceleration of the other. In practice, this is done by assigning to the center of mass of each galaxy a mass-less tracer particle. The orbit of each tracer particle is determined by the smooth gravitational potential associated with the secondary galaxy. If, as in Section 4, a third galactic model is included, the orbit of each tracer particle will be determined by the smooth and non-trivial potential associated with the overlapping density distributions of the two remaining galactic models. Note that, even though we use mass-less particles as phase-space tracers of the galactic centers of mass, we assign to each galactic model a spatially extended density distribution (see Section 3.1.2). Thus, at any given time, the acceleration exerted by the LMC on the MW (and vice versa) is computed by only taking into account the mass that is enclosed within a sphere centered on the LMC (and vice versa) of radius equal to the distance between the two center of masses. In all cases the orbits are integrated backwards from their present-day positions and velocities. 
As in Besla et al. (2007) and K13, we ignore the mass evolution of the LMC owing to the MW's tidal field. In addition, we do not follow the time evolution of the mass or the structural parameters of the MW potential. Since the potentials considered are structurally frozen, there is no dynamical friction exerted on the satellite galaxies. Therefore, we model this acceleration using an approximation of Chandrasekhar's dynamical friction formula (Chandrasekhar 1943; Binney \& Tremaine 2008),

$$
\frac{d \boldsymbol{v}_{2}}{d t}=-4 \pi G^{2} M_{2} \rho_{1} \ln \Lambda\left[\int_{0}^{v_{2}} v^{2} f_{1}(v) d v\right] \frac{\boldsymbol{v}_{2}}{v_{2}^{3}},
$$

towards where the subindex 1 refers to the galaxy causing the friction, the subindex 2 to the galaxy being decelerated, $\boldsymbol{v}_{2}$ is the relative velocity of both interacting galaxies, $M$ is the mass of the corresponding galaxy, $\rho$ the mass density, $f$ the distribution function of velocities, $G$ the gravitational constant and $\Lambda$ is the Coulomb factor. For simplicity, in these experiments we neglect the dynamical friction exerted by the LMC on the MW.

Under the assumption of a Maxwellian velocity distribution and a constant background density field, it is possible to approximate the integral in Equation (1) by:

$$
\int_{0}^{v_{2}} v^{2} f(v) d v \approx\left(\operatorname{erf}(x)-\frac{2 x}{\sqrt{\pi}} e^{-x^{2}}\right)
$$

where $x=v_{2} / \sqrt{2} \sigma$ (Binney \& Tremaine 2008). Here, $\sigma$ is the one-dimensional velocity dispersion of the host DM halo. We adopt an analytic approximation of $\sigma$ for an Navarro-FrenkWhite (NFW) profile as derived by Zentner \& Bullock (2003). Following Besla et al. (2007) and K13, for these experiments we consider a value of the Coulomb factor that varies as a function of the satellite's galactocentric distance as described by Hashimoto et al. (2003). The Hashimoto et al. (2003) Coulomb factor not only scales as a function of the satellite's separation to the host but also as a function of the satellite's scale radius. As in $\mathrm{K} 13$, a fixed scale radius of $3 \mathrm{kpc}$ is assumed in all cases. This may possibly overestimate the role of dynamical friction in the orbital history of high mass LMC models. Detailed comparisons with $N$-body models are required to properly estimate the degree of error, which will be complicated by mass loss owing to MW tides and the presence of the SMC. Such an analysis is beyond the scope of this study. Nonetheless, as we will later show in this section, such effects are very small when considering LMC models that are currently undergoing their first pericenter passage. Moreover, our goal is to assess the effects of a non-inertial frame of reference on the LMC's orbit rather than to determine the exact orbital history itself. As such, this methodology will sufficiently illustrate the general change in the orbits. If indeed dynamical friction is overestimated for high mass LMC models, their orbits will be less eccentric backwards in time, augmenting the perturbative effects we illustrate here.

\subsubsection{Galactic Potentials}

To model the MW potential we choose a three-component system, including a Miyamoto-Nagai disk (Miyamoto \&
Table 1

Parameters of the MW-like Potential Used in Our Simulations

\begin{tabular}{cccccccc}
\hline \hline$M_{\text {vir }}$ & $R_{\text {vir }}$ & $r_{\mathrm{s}}$ & $M_{\text {disk }}$ & $r_{\mathrm{a}}$ & $r_{\mathrm{b}}$ & $M_{\text {bulge }}$ & $c_{\text {bulge }}$ \\
\hline 100 & 261 & 26.47 & 6.5 & 3.5 & 0.53 & 1.0 & 0.7 \\
150 & 299 & 31.27 & 5.5 & 3.5 & 0.53 & 1.0 & 0.7 \\
200 & 329 & 35.15 & 5.0 & 3.5 & 0.53 & 1.0 & 0.7 \\
\hline
\end{tabular}

Note. Masses are in $10^{10} M_{\odot}$ and distances in Kiloparsecs. The scale radius for the Hernquist profile dark matter halos are obtained from $r_{\mathrm{s}}$ through Equation (9).

Nagai 1975)

$$
\Phi_{\text {disk }}=-\frac{G M_{\text {disk }}}{\sqrt{R^{2}+\left(r_{\mathrm{a}}+\sqrt{Z^{2}+r_{\mathrm{b}}^{2}}\right)^{2}}},
$$

a Hernquist bulge (Hernquist 1990),

$$
\Phi_{\text {bulge }}=-\frac{G M_{\text {bulge }}}{r+r_{\mathrm{c}}},
$$

and a NFW DM halo (Navarro et al. 1996)

$$
\Phi_{\text {halo }}=-\frac{G M_{\text {vir }}}{r[\log (1+c)-c /(1+c)]} \log \left(1+\frac{r}{r_{\mathrm{s}}}\right) .
$$

Here, $R$ and $Z$ are the radial and vertical cylindrical coordinates and $r$ is the radial spherical coordinate. The DM halo viral mass, $M_{\text {vir }}$, is defined as the mass enclosed within the radius where the DM density is 360 times the average matter density (van der Marel et al. 2012). In all models the disk scale length and height, $r_{\mathrm{a}}$ and $r_{\mathrm{b}}$, are kept fixed at 3.5 and $0.53 \mathrm{kpc}$, respectively. The bulge mass and scale radius, $M_{\text {bulge }}$ and $r_{\mathrm{c}}$, are also kept fixed at $10^{10} M_{\odot}$ and $0.7 \mathrm{kpc}$, respectively. In addition, the NFW density profiles are truncated at the virial radius. The remaining parameters are allowed to vary in order to explore different models for the MW potential. The adiabatic contraction of the DM halo associated with the presence of a disk was taken into account using the CONTRA code (Gnedin et al. 2004). The values of the parameters for the different models are listed in Table 1 . The circular velocity curve of these Galactic models is shown in Figure 8 of K13. Note that in all cases the circular velocity at the solar circle, $R_{\odot} \approx 8.3 \mathrm{kpc}$, takes a value of $V_{\odot}=239 \mathrm{~km} \mathrm{~s}^{-1}$ (McMillan 2011). Note that a lower value of $V_{\odot}$, e.g., $V_{\odot}=218 \pm 6 \mathrm{~km} \mathrm{~s}^{-1}$ (Bovy et al. 2012), implies a lower mass MW model within its inner regions. Thus, for such Galactic models, any plausible twobody interaction with our LMC models would be enhanced.

The LMC is modeled using a Plummer sphere (Plummer 1911),

$$
\Phi_{\mathrm{LMC}}=-\frac{G M_{\mathrm{LMC}}}{\sqrt{r^{2}+r_{\mathrm{LMC}}^{2}}} .
$$

Following K13, a variety of LMC masses are explored, ranging from $M_{\mathrm{LMC}}=3 \times 10^{10}$ to $2.5 \times 10^{11} M_{\odot}$. A detailed justification for this explored LMC mass range was provided in Section 2. The parameters that describe these LMC models are listed in Table 2 . Note that the scale radius of each model is chosen such that the total mass contained within $9 \mathrm{kpc}$ is $\approx 1.3 \times 10^{10} M_{\odot}$, as indicated by the LMC's rotation curve (van der Marel et al. 2009; van der Marel \& Kallivayalil 2014). 
Table 2

Parameters of the LMC Models Used in Our Simulations

\begin{tabular}{lcccccc}
\hline \hline$M_{\text {LMC }}\left[10^{10} M_{\odot}\right]$ & 3 & 5 & 8 & 10 & 18 & 25 \\
$r_{\text {LMC }}[\mathrm{kpc}]$ & 8 & 11 & 14 & 15 & 20 & 22.5 \\
\hline
\end{tabular}

Note. These parameters are used for both Plummer and Hernquist profiles.

\subsection{An Interacting $M W+L M C$ Model}

\subsubsection{The Orbital Properites of the LMC}

To explore whether artificially fixing the MW's center of mass could have a significant effect on the inferred orbital properties of the LMC, we generate LMC-like orbits by integrating the galaxies backwards in time from their present day phase-space coordinates. The initial orbital conditions for all LMC models, in a Galactocentric reference frame, are $(X, Y, Z)_{\mathrm{LMC}}=(-1,-41,-28) \mathrm{kpc}$ and $\left(v_{x}, v_{y}, v_{z}\right)_{\mathrm{LMC}}=$ $(-57,-226,221) \mathrm{km} \mathrm{s}^{-1}$. The quoted velocities represent the mean value of LMC's velocity and are obtained from K13. Velocities are based on proper motion measurements obtained with three epochs of HST data.

The color-coded dashed lines in Figure 1 show the time evolution of the LMC's galactocentric distance in MW-like models where the center of mass has been artificially nailed down. From top to bottom, the different panels show the results of integrating these orbits over a period of $8 \mathrm{Gyr}$ in Galactic models with DM halo masses of $M_{\text {vir }}=1,1.5$, and $2 \times 10^{12} M_{\odot}$, respectively. As expected, our results are in very good agreement with those found by K13 (see Figure 11 of K13).

In a MW model with $M_{\mathrm{vir}}=1 \times 10^{12} M_{\odot}$ (top panel of Figure 1), the resulting LMC-like orbits show periods larger than a Hubble time, $T_{0} \approx 13.73 \mathrm{Gyr}$, independent of the satellite's total mass. Increasing the mass of our MW models results in shorter LMC orbital periods. As a result, the less massive LMC models start to show more than one pericenter passage within $8 \mathrm{Gyr}$. Note that, as discussed by K13, orbits with periods, $P$, between $T_{0}>P \gtrsim T_{0} / 2 \mathrm{Gyr}$ may not be physical since, according to a general timing argument, the LMC must have had a pericentric approach with the MW very early on, at the time of the Big Bang (Kahn \& Woltjer 1959; Li $\&$ White 2008). Thus, if there has been more than one complete orbit, the LMC period must be $<T_{0} / 2 \approx 6.9$ Gyr. Although this argument suffers from oversimplification, it provides a rough estimate of the largest possible LMC orbital period. In a MW-like host with a mass $M_{\mathrm{vir}}=1.5 \times 10^{12} M_{\odot}$ (middle panel), LMC models with masses $M_{\text {LMC }} \leqslant 5 \times 10^{10} M_{\odot}$ have completed a full orbital period within $6.9 \mathrm{Gyr}$. For our most massive MW model $\left(M_{\mathrm{vir}}=2 \times 10^{12} M_{\odot}\right.$, bottom panel $)$ only the two most massive LMC models $\left(M_{\mathrm{LMC}}=2.5\right.$ and $\left.1.8 \times 10^{11} M_{\odot}\right)$ exhibit orbital periods longer than $6.9 \mathrm{Gyr}$, in agreement with $\mathrm{K} 13$.

The color-coded solid lines in Figure 1 show the orbits of the same LMC models, now in MW potentials that are allowed to freely react to the gravitational pull of the LMC. It becomes abundantly clear that nailing down the MW center of mass has a very significant effect on the backward time integrated orbits, particularly for the most massive LMC models. In all cases, the orbital periods and apocentric distances are significantly shorter. As the mass of the LMC becomes larger, and thus more comparable to the MW mass enclosed within the LMC's location, the two-body interaction becomes more relevant. In other words, the more massive the LMC model, the more significant the changes in the resulting orbits are. This can be inferred from the orbits shown in, e.g., the middle panel of Figure $1\left(M_{\mathrm{vir}}=1 \times 10^{12} M_{\odot}\right)$. The acceleration experienced by the MW toward the LMC, and the corresponding displacement of its center of mass, result in both a shorter LMC orbital period and a smaller apocentric distance in a Galactocentric reference frame.

Note that this change in orbital period is not related to the artificial enhancement of dynamical friction discussed by (White 1983, W83). Using $N$-body simulations, W83 finds that artificially fixing the host's center of mass results in more efficient dynamical friction than when the host is allowed to orbit. The reason for this behavior is attributed to the different global patterns excited by the orbiting satellite on the density distribution of the host (for a detailed discussion about this subject see Cora et al. 1997). As opposed to the $N$-body models considered in W83, the galaxies in our analysis are modeled through analytic and structurally frozen potentials. Thus, the perturbation of the satellite cannot generate wakes in the host's density field. Changes shown in Figure 1 are mainly a reflection of the resulting orbits about the barycenter of the system. Note, however, that due to the shortening of the satellites' orbital periods and apocentric distances, dynamical friction would act more efficiently in the free MW models than in the fixed MW models.

The orbits of the LMC models in a MW-like host with a mass of $1.5 \times 10^{12} M_{\odot}$ are shown in the middle panel of Figure 1. Even in this more massive host, the effects of "freeing" the MW are still very significant. Now, all LMC models with $M_{\mathrm{LMC}} \leqslant 1 \times 10^{11} M_{\odot}$ have completed a full orbit within $6.9 \mathrm{Gyr}$. In a MW-like host with a total mass of $2 \times 10^{12} M_{\odot}$ (bottom panel) all but the most massive LMC model have completed a full orbit within $6.9 \mathrm{Gyr}$.

We summarize and quantify the changes in our LMC-like orbits in Figure 2. The top panel shows the orbital periods of the most recent orbit, obtained in both free (filled symbols) and fixed (open symbols) MW-like models. We focus only on those orbits that have completed at least one orbit about the MW within a Hubble time. This figure clearly shows how dramatic the change on the orbital period can be, especially for the most massive LMC models. For example, for a MW-like host with $M_{\text {vir }}=1.5 \times 10^{12} M_{\odot}$ and a LMC model with $M_{\mathrm{LMC}}=1 \times 10^{11} M_{\odot}$, the period changes from $13.1 \mathrm{Gyr} \approx T_{0}$ (fixed MW) to $6.8 \mathrm{Gyr} \approx T_{0} / 2$ (free $\mathrm{MW}$ ). The bottom panel shows the corresponding changes in the apocentric distance, $R_{\text {apo }}$, as a function of LMC mass. Note that for the MW+LMC mass model combination discussed above, the apocenter goes from $R_{\text {apo }} \approx 1.94 R_{\text {vir }}$ to $R_{\text {apo }} \approx 1.2 R_{\text {vir. }}$. The change in the inferred orbital properties of our LMC-like models suggests that, even though a first-infall is still a very plausible scenario, the limiting LMC-MW mass combinations that could host a first infalling LMC are noticeably affected; it raises the required minimum LMC mass and disfavors MW models with $M_{\text {vir }} \geqslant 1.5 \times 10^{12} M_{\odot}$.

\subsubsection{Phase-space Displacement of the MW Center of Mass}

We have illustrated that the presence of a massive LMC can substantially alter the orbital barycenter of the MW+LMC system even in a first infall scenario. It is thus interesting to 

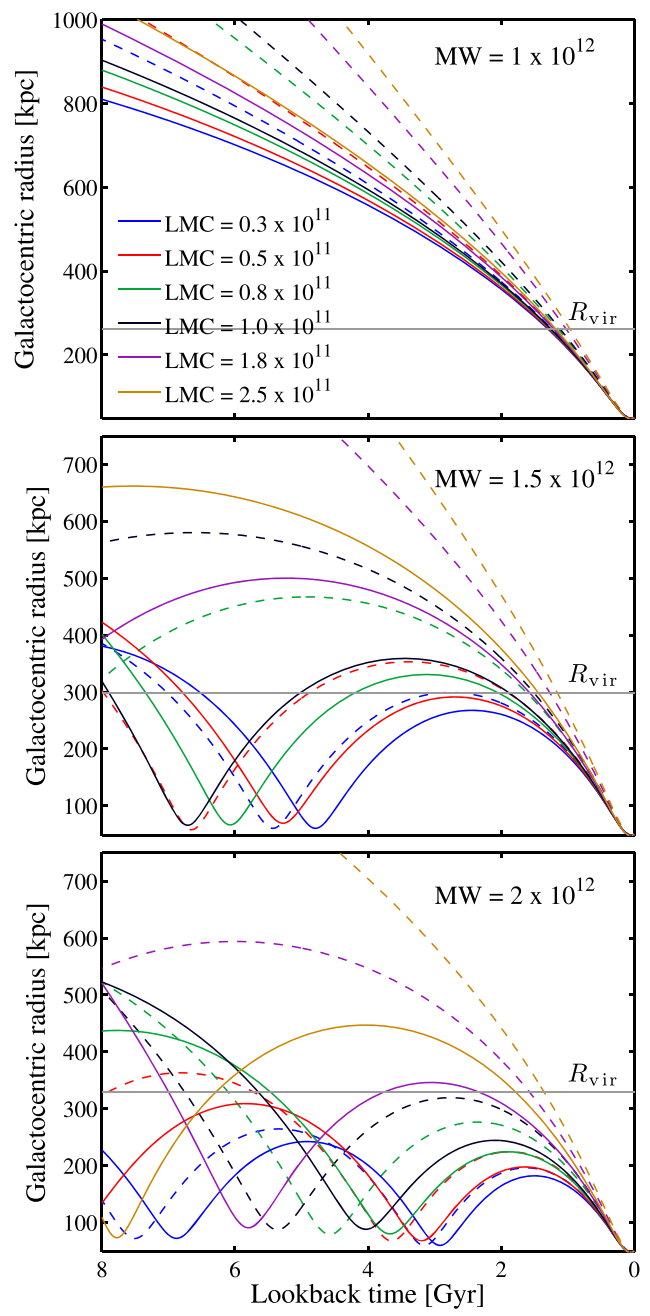

Figure 1. Time evolution of the galactocentric radius of different LMC models in three MW-like host potentials. Orbits are integrated backwards in time. Note that $t=0 \mathrm{Gyr}$ corresponds to present-day. The different color-coded lines show the results obtained with different LMC models, as indicated in the top panel. From top to bottom, the MW models have $M_{\mathrm{vir}}=1,1.5$, and $2 \times 10^{12} M_{\odot}$, respectively. The dashed lines show the results obtained in MW models in which the center of mass has been artificially fixed. Solid lines show the results obtained when the host is allowed to react to the gravitational pull exerted by the LMC. Note the shorter LMC orbital periods obtained in the latter case. The more massive the LMC, the larger the change in orbital period.

quantitatively characterize the displacement of the MW center of mass as function of time due to this gravtiational interaction.

Before we move any forward, it is worth recalling that the inferred LMC's orbital properties, quoted both here and in $\mathrm{K} 13$, do suffer from a number of simplifications. If the LMC orbits are significantly affected by this simplifications, then the estimated phase-space displacement of the MW center of mass could also be significantly affected.

On the one hand, as shown in K13, including a simple model for the time evolution of the MW potential would increase these orbital periods by $\lesssim 2 \mathrm{Gyr}$ (the exact amount depends on the mass of the host and the satellite). In addition, we have only considered the orbits associated with the mean LMC velocity presented in K13. The relatively large uncertainty on each of the velocity components, of $\approx \pm 19 \mathrm{~km} \mathrm{~s}^{-1}$, will yield, in some cases, orbits with significantly larger periods. Furthermore, we have neglected LMC mass loss due to the tidal interaction with the MW. Note, however, that perturbations on the inferred
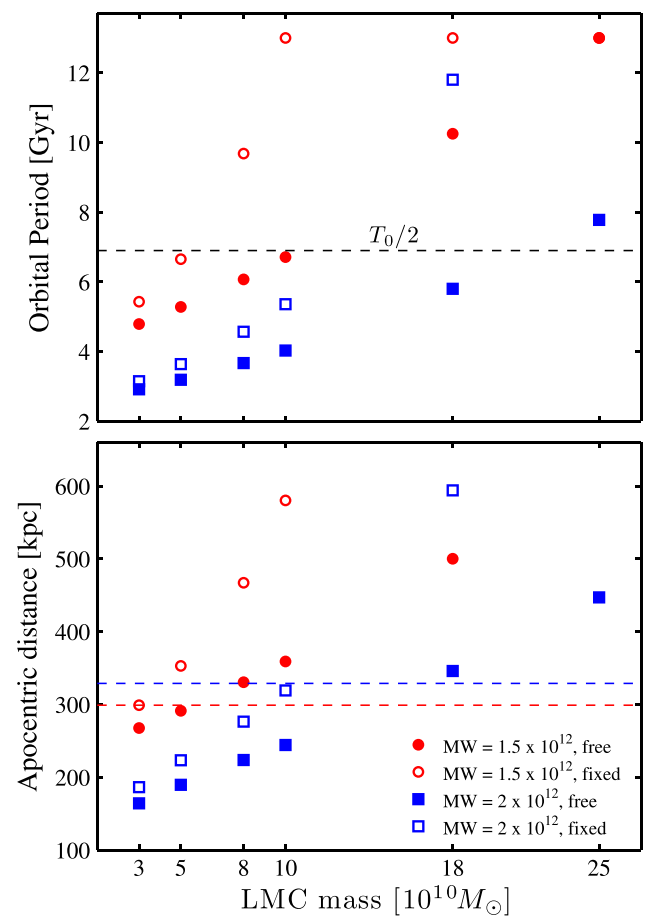

Figure 2. Top panel: orbital period of LMC-like orbits as a function of the LMC total mass. The red and blue symbols indicate the period of orbits integrated in MW-like host with $M_{\text {vir }}=1.5$ and $2 \times 10^{12} M_{\odot}$, respectively. Open symbols show the orbital periods obtained when the MW is artificially fixed in space. Filled symbols show the results obtained when the MW is allowed to freely react to the gravitational pull exerted by the LMC. The black dashed line indicates half of the Hubble time, $T_{0} / 2$. Bottom panel: as in the top panel, for the apocentric distance of the different LMC-like orbits. The red and blue dashed lines indicate the virial radius of the MW-like host with $M_{\mathrm{vir}}=1.5$ and $2 \times 10^{12} M_{\odot}$, respectively. The orbital properties shown in this figure were obtained using the mean LMC's velocity presented in K13.

orbital properties due to LMC mass loss are not expected to be significant in those cases where the LMC is clearly undergoing its first infall. On the other hand, the treatment of dynamical friction implemented here may be overestimated, thus artificially increasing the orbital periods (see Section 3.1.1).

A more accurate determination of the LMC's orbital properties as a function of LMC mass would require a full $\mathrm{N}$-body treatment. Even though this is not the goal of this work, to explore whether our approximations regarding dynamical friction and LMC's mass loss are valid, we have ran a full $\mathrm{N}$ body simulations considering one of the MW+LMC mass combinations analyzed in this work. The MW-like host was modeled as a self-consistent three-component system consisting of a NFW DM halo, an exponential stellar disk, and a central bulge following a Hernquist profile. The LMC galaxy was modeled as a self-consistent Plummer sphere. The masses and parameters that specify each galactic component were chosen to reproduce the analytic rigid representation of the galactic potentials associated with a MW of $M_{\text {vir }}=1 \times 10^{12} M_{\odot}$ and a LMC of total mass $M_{\mathrm{LMC}}=1.8 \times 10^{11} M_{\odot}$. These parameters are listed in Table 3. Initial positions and velocities for the LMC and MW centers of mass were obtained from the numerically integrated orbits using the analytic rigid potentials. The simulations were started at a lookback time equal to $2 \mathrm{Gyr}$.

In Figure 3 we show, with purple lines, the time evolution of the position (top panel) and the velocity (bottom panel) of the 

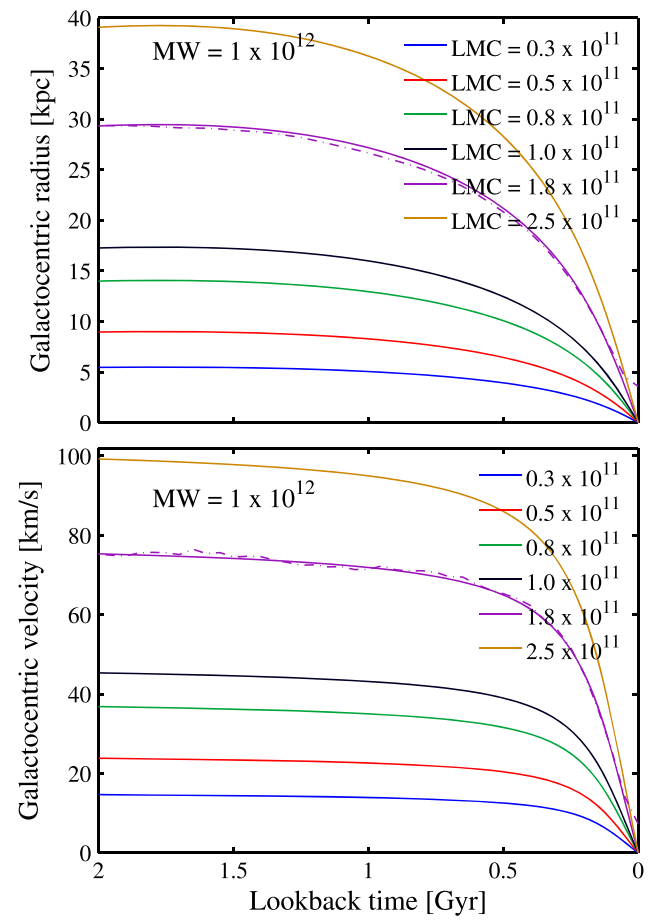

Figure 3. Time evolution of the position and velocity of the MW center of mass with respect to its position at $t=0 \mathrm{Gyr}$. The results are obtained from simulations where the MW model is allowed to react to the gravitational pull exerted by the LMC. Orbits are integrated backwards in time. Note that $t=0 \mathrm{Gyr}$ corresponds to present-day. The solid lines show the results obtained with a MW model with a dark matter halo of $M_{\text {vir }}=1 \times 10^{12} M_{\odot}$. The different colors indicate the results obtained with different LMC models, as indicated in the top right corner of the top panel. The most significant changes in both the position and the velocity of the MW center of mass take place only during the last $0.3-0.5 \mathrm{Gyr}$, the time at which both, the MW mass enclosed within the LMC Galactocentric radius becomes comparable to that of the LMC and the distance between both galaxies becomes short enough. For comparison, the dashed-dotted line shows the displacement of the MW center of mass obtained from a fully live $N$-body simulations considering an LMC model with $M_{\mathrm{LMC}}=1.8 \times 10^{11} M_{\odot}$. Note the very good agreement between the results obtained with the rigid analytic potential and their fully live $N$-body counterpart.

MW center of mass, with respect to its present-day coordinates, due to the gravitational interaction with the LMC model $M_{\mathrm{LMC}}=1.8 \times 10^{11} M_{\odot}$. The solid line show the result from the rigid analytic representation of the potentials while the dashed-dotted line shows the result from for the fully $\mathrm{N}$-body representation. Note the very good agreement between the results obtained from the two different modeling techniques. The final phase-space displacement of the MW center of mass in the $\mathrm{N}$-body model is slightly smaller than what was obtained with the analytic rigid case. The differences are $\approx 4 \mathrm{kpc}$ and $\approx 7$ $\mathrm{km} \mathrm{s}^{-1}$ in position and velocity, respectively. Furthermore, we find the final location of the LMC center of mass in the $N$-body model to be $4 \mathrm{kpc}$ further away from the MW center of mass than its observationally constrained Galactocentric distance. Thus, it is likely that by selecting a more suitable set of ICs for the $N$-body simulations, such that in these calculations the LMC center of mass finish at the desired location, this (already small) discrepancy could even become smaller.

We conclude that our approach of using rigid, spatially extended density distributions to compute the orbital evolution of the LMC and MW is an adequate approximation for this study. With this in mind, for the remainder of this section we
Table 3

Summary of the Set-up for the $N$-body Simulations Analyzed in Section 5

\begin{tabular}{|c|c|c|c|}
\hline Host & & & \\
\hline DM halo & & & $N_{\text {part }}=2.65 \times 10^{5}$ \\
\hline Virial mass & $1 \times 10^{12}$ & & {$\left[M_{\odot}\right]$} \\
\hline Scale radius & 26.5 & & {$[\mathrm{kpc}]$} \\
\hline Concentration & 9.86 & & \\
\hline Stellar disk & & & $N_{\text {part }}=3 \times 10^{4}$ \\
\hline Mass & $6.5 \times 10^{10}$ & & {$\left[M_{\odot}\right]$} \\
\hline Scale length & 3.5 & & {$[\mathrm{kpc}]$} \\
\hline Scale height & 0.53 & & {$[\mathrm{kpc}]$} \\
\hline Stellar bulge & & & $N_{\text {part }}=5 \times 10^{3}$ \\
\hline Mass & $1 \times 10^{10}$ & & {$\left[M_{\odot}\right]$} \\
\hline Scale radius & 0.7 & & {$[\mathrm{kpc}]$} \\
\hline Sgr Satellites & & & \\
\hline DM halo & Light & Heavy & $N_{\text {part }}=2.65 \times 10^{4}$ \\
\hline Virial mass & $0.32 \times 10^{11}$ & $1 \times 10^{11}$ & {$\left[M_{\odot}\right]$} \\
\hline Scale radius & 4.9 & 6.5 & {$[\mathrm{kpc}]$} \\
\hline Stellar spheroid & & & $N_{\text {part }}=5 \times 10^{4}$ \\
\hline Mass & $6.4 \times 10^{8}$ & $6.4 \times 10^{8}$ & {$\left[M_{\odot}\right]$} \\
\hline Scale radius & 0.85 & 0.85 & {$[\mathrm{kpc}]$} \\
\hline LMC Satellite & & & \\
\hline Single spheroid & & & $N_{\text {part }}=2 \times 10^{4}$ \\
\hline Mass & $1.8 \times 10^{11}$ & & {$\left[M_{\odot}\right]$} \\
\hline Scale radius & 20 & & {$[\mathrm{kpc}]$} \\
\hline
\end{tabular}

will only consider rigid analytic potentials. In Figure 3, different colored lines indicate the time evolution of the position and the velocity of the MW center of mass induced by different LMC-like models. In all cases, the MW model corresponds to that with $M_{\mathrm{vir}}=1 \times 10^{12} M_{\odot}$. Note that, for this low-mass MW model, the LMC is on its first infall about the MW, regardless of the LMC mass.

Changes in both position and velocity are very rapid and take place primarily during the last $\approx 0.3-0.5 \mathrm{Gyr}$. As the LMC approaches its present-day position, $R_{\mathrm{LMC}} \approx 50 \mathrm{kpc}$, the mass of the MW enclosed within a radius of $R_{\mathrm{LMC}}$ becomes smaller. Assuming a MW model with $M_{\mathrm{vir}}=1 \times 10^{12} M_{\odot}$, at present day $M_{\mathrm{MW}}^{50} \approx 3.7 \times 10^{11} M_{\odot}$ becomes comparable to the mass of the LMC. Thus, the orbital barycenter of the MW+LMC system is significantly displaced from the MW center of mass. For example, for a LMC model with a total mass $M_{\mathrm{LMC}}=1.8 \times 10^{11} M_{\odot}$, the orbital barycenter is located at $\approx 14 \mathrm{kpc}$ from the MW center of mass at the present epoch. In this model, as the LMC approaches its current location the MW is displaced by $\approx 30 \mathrm{kpc}$ and its velocity has changed by $\approx 75$ $\mathrm{km} \mathrm{s}^{-1}$ in $\approx 0.5 \mathrm{Gyr}$.

Interestingly, we find very similar phase-space displacements in our more massive MW models. The circular velocity at the location of the Sun limits the amount of mass that can exist in the DM halo at small radii. As such, to create more massive MW models, the bulk of the mass is added at radii beyond $50 \mathrm{kpc}$. Correspondingly, the virial radii of the halos increase. This means that the resulting mass enclosed within $50 \mathrm{kpc}$ is not strikingly different in the three MW models we adopt. 
The results presented in this section highlight the importance of self-consistent modeling of the MW and LMC interaction when trying to constrain the LMC's orbital properties. Given the magnitude of the effect of this interaction on the motion of the MW, it is of interest to explore its implications for stars in the MW stellar halo. Owing to the extended nature of the stellar halo, not all stars will be accelerated at the same rate. Thus, this may affect the observable properties of spatially extended stellar streams.

\section{THE ORBIT AND TIDAL DEBRIS FROM SGR}

In this section we explore the implications of the motion of the MW around its center of mass with the LMC for the orbit of the Sgr and its distribution of tidal debris. For this purpose, we will integrate Sgr-like orbits in different scenarios. We will consider spatially "free" and "fixed" MW-like hosts, with and without the presence of a LMC-like satellite. As in Section 3, the experiments analyzed in this section will assume smooth and analytic representations of the Galactic potentials. The models and methodology are described in Section 4.2. We briefly review the main properties of the Sgr stream and discuss previous attempts to constrain the shape of the MW DM halo based on the stream's phase-space distribution in Section 4.1. Our results are presented on Section 4.3. Note that, throughout this study, we are not interested in obtaining an orbit that could accurately reproduce the observed distribution of the Sgr tidal debris. Our goal is rather to simply characterize the significance of artificially fixing the MW center of mass on Sgr-like orbits. If the effect is significant, this justifies the incorporation of complete and realistic modeling of the LMC+MW interaction in future analyzes of Sgr and other long stellar streams in the halo of the MW.

\subsection{The Complex Nature of the Sgr Streams}

As discussed in Section 1, the Sgr tidal stream and its remnant core have been used multiple times in the past to probe the mass distribution of the MW. The main reason behind the wide popularity of this satellite galaxy is the very large radial and angular extent covered by its debris. The Sgr stellar stream spans at least $300^{\circ}$ across the sky (Ibata et al. 1997), and observations suggest that it can be observed at Galactocentric distances as large as $100 \mathrm{kpc}$ (e.g., Majewski et al. 2003; Newberg et al. 2003; Ruhland et al. 2011; Drake et al. 2013). As discussed by Deg \& Widrow (2014), the Sgr stream has a very complicated structure, making it difficult to model. The mean orbital poles of the great circles that best fit debris leading and trailing the Sgr core show a difference of $\sim 10^{\circ}$ (Johnston et al. 2005). Stars in the trailing and leading arms show very different apocenters (Belokurov et al. 2014) and bifurcations have been observed in both arms (Belokurov et al. 2006; Koposov et al. 2012; Slater et al. 2013). In addition, Peñarrubia et al. (2010) showed that the phase-space configuration of the Sgr stream strongly depends on the structure of the progenitor.

Given the complex nature of this stream, it is not surprising that several previous studies have yielded contradictory results with regards to the structure of the MW's gravitational potential. For example, the previously mentioned tilt of the orbital plane can be reproduced with $N$-body simulations if the MW DM halo is modeled as a mildly oblate galactic component (Johnston et al. 2005). On the other hand, radial velocity measurements of a sample of $M$ giant stars favor a prolate DM halo (Helmi 2004). Furthermore, Law \& Majewski (2010) showed that a triaxial DM halo model could reproduce the angular position, distance, and radial velocity constraints imposed by current wide-field surveys of the Sgr stream. However, the results from this model are bound by a number of caveats. First, the model requires the disk's minor axis to be aligned with the intermediate axis of the triaxial halo. As shown by Debattista et al. (2013), this configuration is extremely unstable. The problem can be alleviated if the assumption of a disk-halo alignment is relaxed when searching for a best fitting Galactic potential (Deg \& Widrow 2014). second, the resulting axis ratios are not compatible with expectations derived from cosmological simulations (e.g., see discussion by Vera-Ciro \& Helmi 2013, hereafter VCH13). Interestingly, $\mathrm{VCH} 13$ showed that if the gravitational field of the LMC is taken into account when computing the orbit of Sgr, the triaxial configuration of the MW-like DM halo can be brought to a more cosmologically plausible shape.

The analysis presented in $\mathrm{VCH} 13$ shows that the torque on Sgr exerted by the LMC can be as important as that of the MW's DM halo, introducing non-negligible perturbations to the orbit of Sgr and its distribution of debris. Attempts to reproduce the Sgr stream without a model for the LMC perturbation will consequently force searches of the best fitting parameters that characterize the MW's gravitational potential to artificially adjust in order to account for this perturbation.

While a very relevant conclusion, the work of VCH13 (as well as many of the previously cited works) considered MW models that are fixed in phase-space. In addition, their results were based on test particle simulations in which the Sgr stream is being significantly perturbed by the LMC over 3-4 Gyr. As shown in Section 3, even relatively low-mass first-infall LMC models can significantly accelerate the MW inner regions in a very short period of time. It is thus likely that, in a Galactocentric reference frame (as opposed to a barycentric reference frame), the distribution of Sgr debris, which covers a radial extension of $\sim 100 \mathrm{kpc}$, will be significantly perturbed due to the phase-space displacement of the MW center of mass. To explore this, we integrate Sgr-like orbits in MW potentials that are allowed to freely react to the gravitational pull of the LMC.

\subsection{Analytic Models}

\subsubsection{Methodology}

As in Section 3, to follow the gravitational interaction between the MW, the LMC, and Sgr, we used a symplectic leapfrog integration scheme (Springel et al. 2001). The host and the two satellites are represented with analytic potentials; the center of each one follows the orbit that results from the acceleration of the other two. In all cases the orbits are integrated backwards in time from their present-day positions and velocities. For simplicity, in this section we model the DM halo of all galaxies with Hernquist profiles. This allows us to model the dynamical friction that each of our three galaxies (LMC, Sgr, and MW) induces on the remaining two by approximating the integral in Equation (1) as follows:

$$
\int_{0}^{v_{2}} v^{2} f(v) d v \approx \frac{1}{6}\left(\operatorname{erf}(x)-\frac{2 x}{\sqrt{\pi}} e^{-x^{2}}\right),
$$

where $x=2 v_{2} \sqrt{r_{1} /\left(M_{1} G\right)}$. Here, $r_{1}$ is the scale radius of the 
galaxy causing the friction. The Coulomb factor is computed as

$$
\Lambda=\frac{r v_{2}^{2}}{G M_{2}(r)},
$$

where $r$ is the distance between the centers of the two galaxies and $M_{2}(r)$ is the mass of the galaxy being decelerated enclosed within $r$ (Carpintero et al. 2013).

\subsubsection{Galactic Models}

We model the MW as a three component system. The main difference with the MW model presented in Section 3.1.2 is the profile of its DM halo. In this Section, all DM halos follow a Hernquist profile (Equation (4)). The parameters that describe our MW models are listed in Table 1 . The scale radius of the Hernquist profile DM halos, $r_{\mathrm{H}}$, are obtained from the NFW scale radii listed in Table 1 following Springel et al. (2005),

$$
r_{\mathrm{H}}=r_{\mathrm{s}} \sqrt{2\left(\log (1+c)-\frac{c}{1+c}\right)} .
$$

To smoothly model Sgr, we also choose a Hernquist profile. The model parameters are based on those presented by (Purcell et al. 2011, hereafter P11). Our single component model consists of a DM halo with a mass, prior to crossing the MW virial radius $\left(R_{\mathrm{vir}}\right)$, of $M_{\mathrm{Sgr}}=10^{11} M_{\odot}$. As described by $\mathrm{P} 11$, this large value of $M_{\text {Sgr }}$ prior to infall is obtained from a cosmological abundance matching argument (Conroy \& Wechsler 2009; Behroozi et al. 2010), based on the presentday luminosity of the Sgr core and tidal debris. Lower mass models of Sgr are presented in Section 5. Note however that, as in P11 and references therein, the satellite is initially launched 2 Gyr ago at a distance of $80 \mathrm{kpc}$ from the Galactic center, traveling vertically at $80 \mathrm{~km} \mathrm{~s}^{-1}$ toward the North Galactic Pole. Thus its mass, at this point in time, is truncated at the instantaneous Jacobi radius $r_{\mathrm{J}} \approx 30 \mathrm{kpc}$. This leaves a total bound mass $\left(2 \mathrm{Gyr}\right.$ ago) of $M_{\mathrm{Sgr}}^{80} \approx 3.8 \times 10^{10} M_{\odot}$, i.e., a factor of $\sim 3$ smaller than its effective virial mass at infall, $M_{\mathrm{vir}}$. The scale length of the profile is $R_{\mathrm{Sgr}}=13 \mathrm{kpc} .{ }^{10}$ In order to crudely account for Sgr galaxy's mass loss due to tidal interaction with the MW potential, we assume that its mass linearly varies during the $2 \mathrm{Gyr}$ of evolution between $M_{\mathrm{Sgr}}^{80}$ and $M_{\text {Sgr }}=10^{9} M_{\odot}($ Law et al. 2005; Purcell et al. 2011). A Hernquist profile is also used to model the LMC. The parameters that specify each of our LMC models are listed in Table 2.

\subsection{An Analytic Treatment of the $M W+L M C+S g r$ System}

In this Section we characterize the significance of the perturbative effects associated with a first-infall LMC on the orbit of Sgr. In all experiments, the orbits of our three galaxies are integrated backward in time for $2 \mathrm{Gyr}$ from their presentday positions. To be consistent with the full $N$-body integrations that we analyze in Section 5, present-day ICs for our Sgr-like orbits are obtained as follows. As discussed in Section 4.2.2, we first integrate our Sgr model forward in time for $2 \mathrm{Gyr}$ in a free MW model. The ICs at this initial time are $(X, Y, Z)_{\mathrm{Sgr}}=(80,0,0) \mathrm{kpc}$ and $\left(v_{x}, v_{y}, v_{z}\right)_{\mathrm{Sgr}}=(0,0,80) \mathrm{km} \mathrm{s}^{-1}$. Note that, only in this first step, we neglect Sgr's mass loss due

\footnotetext{
${ }^{10}$ Note that the equivalent NFW scale radius is $6.5 \mathrm{kpc}$ (see Equation 9).
}
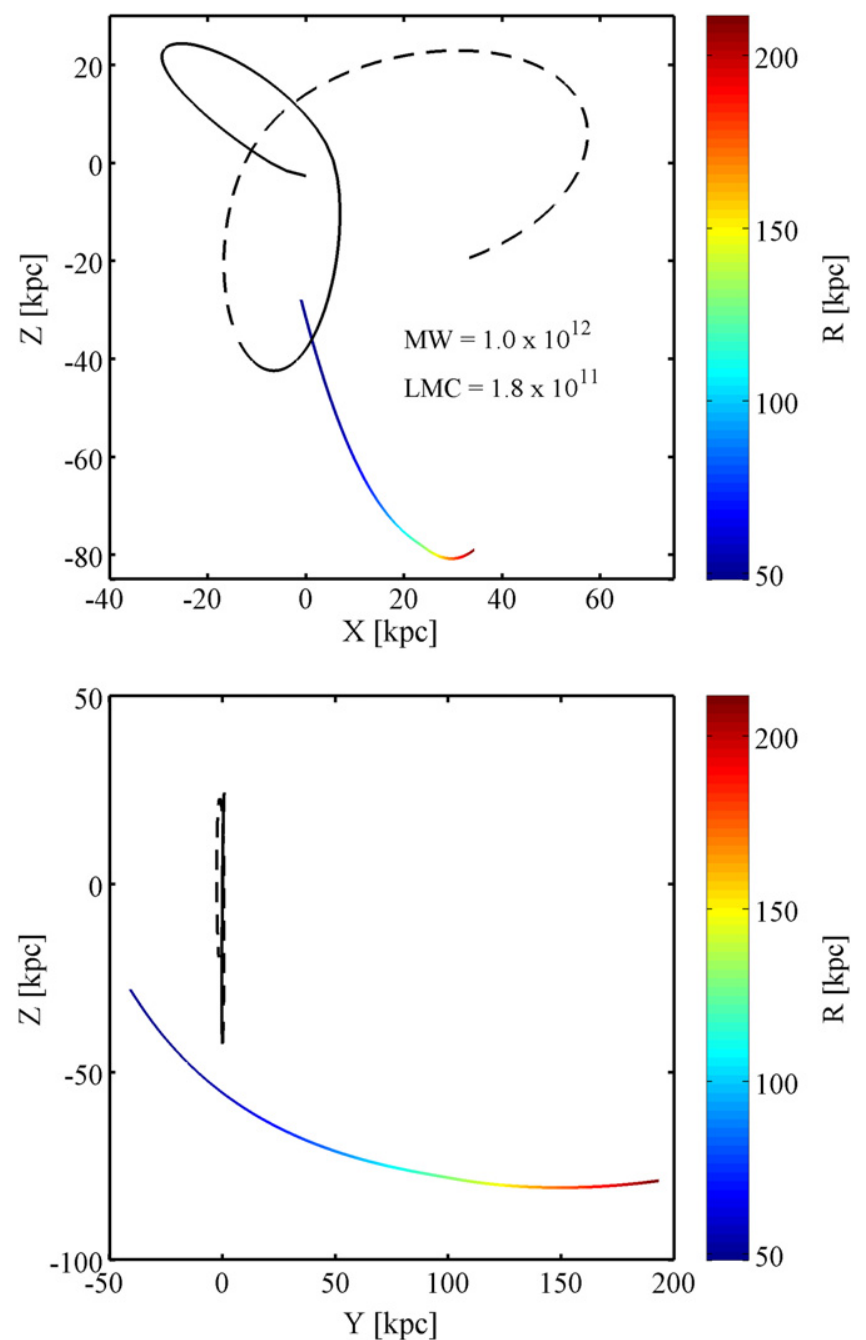

Figure 4. Sgr-like and LMC orbits obtained in a free MW model with a dark matter halo of $10^{12} M_{\odot}$. For this example an LMC model with total mass $M_{\mathrm{LMC}}=1.8 \times 10^{11} M_{\odot}$ was considered. The orbits are illustrated in the $X Z$ (top panel) and $Y Z$ (botom panel) Galactocentric planes, with $Z$ pointing toward the Galactic pole and $X$ pointing toward the opposite direction of the Sun. The black and color coded lines indicate the Sgr-like and the LMC orbit, respectively. The color coding indicates the LMC's galactocentric distance. Solid and dashed black lines show the Sgr's first and the second Gyr of backwards evolution, respectively. For clarity, the LMC orbit is only shown during the first Gyr of backwards evolution.

to the tidal interaction with the MW potential. The position and velocity of the Sgr model at the final integration point (i.e., after 2 Gyr of evolution), $(X, Y, Z)_{\mathrm{Sgr}}=(0,0,-3) \mathrm{kpc}$ and $\left(v_{x}, v_{y}, v_{z}\right)_{\mathrm{Sgr}}=(413,0,-46) \mathrm{km} \mathrm{s}^{-1}$, are then used as presentday ICs for the backward integration. As an example, we show in Figure 4 the resulting Sgr-like and LMC orbits obtained in a free MW model with a DM halo of $10^{12} M_{\odot}$. For this integration an LMC model with total mass $M_{\mathrm{LMC}}=1.8 \times 10^{11} M_{\odot}$ was considered.

In Figure 5, we compare the resulting backward integrated Sgr-like orbits obtained with free and fixed MW models, with and without the LMC. The blue line in the left panel shows the backward time integrated Galactocentric orbit of our Sgr-like satellite in a MW model with a DM halo of $10^{12} M_{\odot}$. The solid and dashed lines indicate the first and second Gyr of evolution, respectively. In this orbital integration, the MW center of mass is allowed to react to any external potential. However, the 


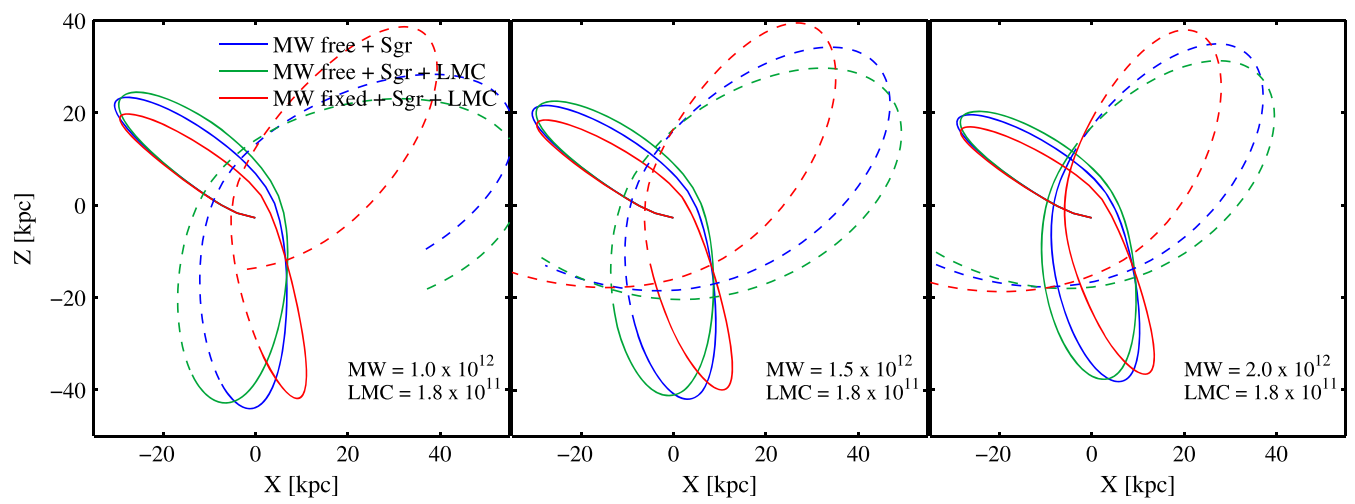

Figure 5. Different lines show Sgr-like orbits integrated for 2 Gyr in different models of the MW potential. The orbits are illustrated in the $X Z$ Galactocentric plane, with $Z$ pointing toward the Galactic pole and $X$ pointing toward the opposite direction of the Sun. The LMC moves in a direction that is approximately perpendicular to this plane. Sgr orbits are integrated backwards in time. Thus, present-day positions are the same in all cases. Solid and dashed lines show the first and the second Gyr of backwards evolution, respectively. The blue lines show the Sgr-like orbits obtained in MW models that are allowed to react to any external perturbation (Sgr+MW only). The green lines show the same orbits, now introducing a LMC model with a mass of $M_{\mathrm{LMC}}=1.8 \times 10^{11} M_{\odot}$, which follows the orbit described in Figure 1 . The red lines show the corresponding Sgr orbit obtained after artificially fixing the MW center of mass, but including the perturbative effects of the LMC. From left to right, the different panels show the results obtained using MW models with masses $M_{\mathrm{vir}}=1,1.5$, and $2 \times 10^{12} M_{\odot}$, respectively. Note that the differences on the Sgr-like orbits between "free" (green line) and "fixed" (red line) MW models are even larger that those obtained in models with and without the LMC (green and blue lines).

initial mass of Sgr, $10^{9} M_{\odot}$, is very small and thus the orbital barycenter is approximately located at the MW center of mass.

The green line in the same panel shows the orbit of Sgr in a Galactocentric reference frame, now including a model for the LMC. As before, we allow the MW center of mass to react to the pull of any external potential. For this experiment we have chosen a LMC model with a total mass of $M_{\mathrm{LMC}}=1.8 \times 10^{11} M_{\odot}$ that is experiencing its first pericentric passage at the present-day (see Section 3 and Table 2). This LMC model represents the canonical model described by K13. Such a massive LMC is required to keep the LMC-SMC binary configuration for longer than $2 \mathrm{Gyr}$ in MW models with masses $\leqslant 1.5 \times 10^{12} M_{\odot}$ (Gnedin et al. 2010; Boylan-Kolchin et al. 2013; Kallivayalil et al. 2013; Piffl et al. 2014). It has also been used in the past to successfully reproduce many of the observable properties of the Magellanic stream (Besla et al. 2012). A comparison of the blue and green lines shows that the perturbation on the Sgr orbit due to the LMC's gravitational pull is indeed significant, as first suggested by VCH13. Note, however, that in our case we are considering a first infall scenario for the LMC, and so its perturbative effects have only operated over the past $\sim 1.5 \mathrm{Gyr}$.

As indicated by the solid lines, the Sgr orbital perturbation is significant even within the past $1 \mathrm{Gyr}$ of backwards evolution. With a red line we now show the orbit of our Sgr model in a Galactocentric reference frame, including the same LMC model but, as in VCH13, keeping the MW center of mass fixed at all times. Note the very different orbit for Sgr that is obtained when the MW is not allowed to react to the gravitational pull exerted by its satellites, especially the LMC. The differences in the Sgr-like orbits between a "free" (green line) and a "fixed" (red line) MW model are even larger that those obtained in models with (green) and without (blue) the LMC.

Another noticeable effect is the different angular distances between the last two apocenters of the green and red orbits, $\Theta_{\text {apo }}$. As shown in Figure 6, allowing the MW model to react to the pull of its satellites, especially the LMC, results in a significant decrease of $\Theta_{\text {apo. }}$. A comparison between the Sgr-like orbits in the "free" and the "fixed" MW models that include the
LMC yields a $\Delta \Theta_{\text {apo }} \approx 17^{\circ}$. Instead, as can be seen in the left panel of Figure 5, a comparison between the Sgr orbits obtained in free MW models with (green line) and without the LMC (blue line) yields a smaller but still noticeable $\Delta \Theta_{\text {apo }} \approx 8^{\circ}$. (Belokurov et al. 2014, hereafter B14) finds a $\Theta_{\text {apo }}$ between the apocenters of the Sgr leading and trailing arms that is $\sim 25^{\circ}$ smaller than what is predicted for Sgr orbits in logarithmic fixed halos. Thus, taking into account a free MW and a model of the LMC could at least partially explain this observed smaller-than-predicted angular distance between the consecutive apocenters. Note that the magnitude of $\Delta \Theta_{\text {apo }}$ strongly depends on the initial orbital conditions of Sgr, as well as on the mass of the three galaxies involved. The middle and right panels of Figure 5 show the same experiments, now in MW models with 1.5 and $2 \times 10^{12} M_{\odot}$, respectively. Even in these more massive MW models the perturbation to the orbit of Sgr associated with fixing the MW center of mass is very significant, and again larger than that obtained by the inclusion of the LMC torques on the Sgr orbit alone.

In Figure 7 we explore the Sgr orbital history including LMC models of different total masses. In all cases, the MW model contains a DM halo of $1 \times 10^{12} M_{\odot}$. The left panel shows the results obtained with our least massive LMC model, $M_{\mathrm{LMC}}=3 \times 10^{10} M_{\odot}$. In this case, perturbations to the orbit of Sgr are almost negligible, regardless of whether we include a model for the LMC or consider a free MW. However, as we increase the mass of the LMC, the perturbation on the orbit of Sgr quickly becomes noticeable. For a LMC with $M_{\mathrm{LMC}}=8 \times 10^{10} M_{\odot}$ (i.e., the mass used by $\mathrm{VCH} 13$ ), the perturbation is very clear. As before, the largest changes in the orbital path of Sgr are obtained when we allow the MW to react to the external gravitational potential of the LMC.

\section{5. $N$-BODY MODELS OF THE PHASE-SPACE DISTRIBUTION OF SGR-LIKE TIDAL DEBRIS}

Thus far, we have explored the effects of allowing the MW's center of mass to respond to perturbations from its satellites on the history of the Sgr dwarf galaxy's orbit. However, such analytic arguments are insufficient to explore the significance 


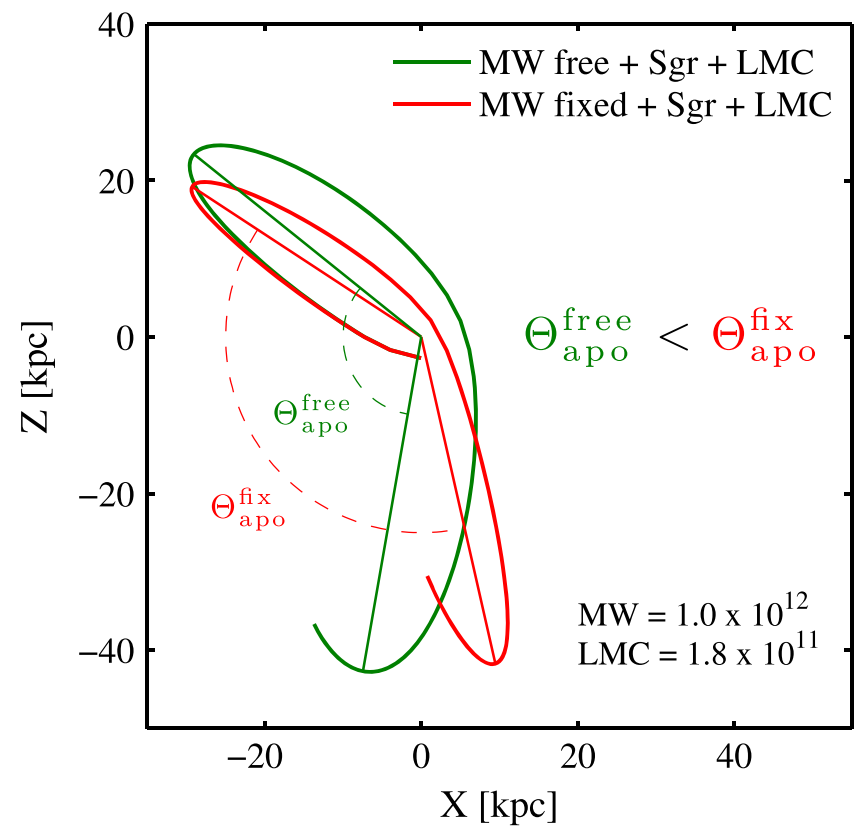

Figure 6. Angular distance between two consecutive apocenters obtained from Sgr-like orbits integrated in a free (green) and a fixed (red) MW model. In both cases, a model for the LMC is also included in the simulations. For clarity, orbits are shown only during the first Gyr of backwards evolution. Note that allowing the MW model to react to the pull of its satellites, especially the LMC, results in a significant decrease of $\Theta_{\text {apo }}$.

of such perturbations on the phase-space distribution of the Sgr stellar stream. To explore this, we run a new set of experiments, now based on full $N$-body numerical simulations following the evolution of Sgr forward in time. We describe the models and methodology in Section 5.1, and present our results in Section 5.2.

\subsection{N-body Models}

\subsubsection{Methodology}

The $N$-body systems are evolved using GADGET-2.0 (Springel 2005), a well-documented, massively parallel TreeSPH code. To construct self-consistent stable models of the MW, the LMC, and Sgr, we follow the procedure described by Villalobos \& Helmi (2008). In the following sections we describe the main properties of each galactic model. In general, the force softening is chosen to be a tenth of the mean interparticle distance of each system, calculated using particles located within a distance of ten scale length radii. In particular, when dealing with Plummer models we compute our softening lengths as described by Athanassoula et al. (2000).

Keeping a MW model fixed in a $N$-body simulation is significantly more challenging than in simulations with analytic, smooth galactic models. Having illustrated in Section 4.3 that perturbations on the orbit and debris of Sgr when fixing the MW are quite significant, in what follows we will only consider free MW models with and without the presence of a massive LMC. Note that, due to the relatively low mass of Sgr, the pertubative effects associated with the phasespace displacement of the MW center of mass in previous studies that have only considered the interaction between the MW and Sgr (i.e., disregarding the LMC) are negligible.

\subsubsection{Galactic Models}

We model the MW-like host as a self-consistent threecomponent system containing a NFW DM halo, an exponential stellar disk, and a central bulge following a Hernquist profile. The DM halo has a total mass of $M_{\mathrm{vir}}=10^{12} M_{\odot}$, a scale radius $r_{\mathrm{s}}=26.47 \mathrm{kpc}$, and is initially adiabatically contracted to model its response to the formation of a stellar disk in its central region (Blumenthal et al. 1986; Mo et al. 1998). The exponential disk has a total mass of $6.5 \times 10^{10} M_{\odot}$ and a scale length and height of 3.5 and $0.53 \mathrm{kpc}$, respectively. For the bulge we assume a mass of $10^{10} M_{\odot}$ and a scale radius of $0.7 \mathrm{kpc}$. As previously discussed, the circular velocity profile takes a value of $\sim 239 \mathrm{~km} \mathrm{~s}^{-1}$ at $\sim 8.29 \mathrm{kpc}$ from the galactic center.

We use a Plummer distribution to model the LMC. For this set of numerical experiments we consider a profile with a total mass of $M_{\mathrm{LMC}}=1.8 \times 10^{11} M_{\odot}$ and a scale radius $r_{\mathrm{LMC}}=20$ kpc. Since the mass spreads out to infinity in Plummer models, the density profile is initially truncated at the radius that encloses $95 \%$ of the LMC's total mass.

Based on Purcell et al. (2011), the Sgr progenitor is selfconsistently initialized with a NFW DM halo and a spheroidal stellar component that follows a Hernquist profile. Two different DM mass models are considered:

1. a "Light" model with a DM halo mass of $10^{10.5} M_{\odot}$;

2. a "Heavy" model with a DM halo mass of $10^{11} M_{\odot}$.

The stellar components in both models have a total mass of $6.4 \times 10^{8} M_{\odot}$ and a scale radius of $0.85 \mathrm{kpc}$ (Niederste-

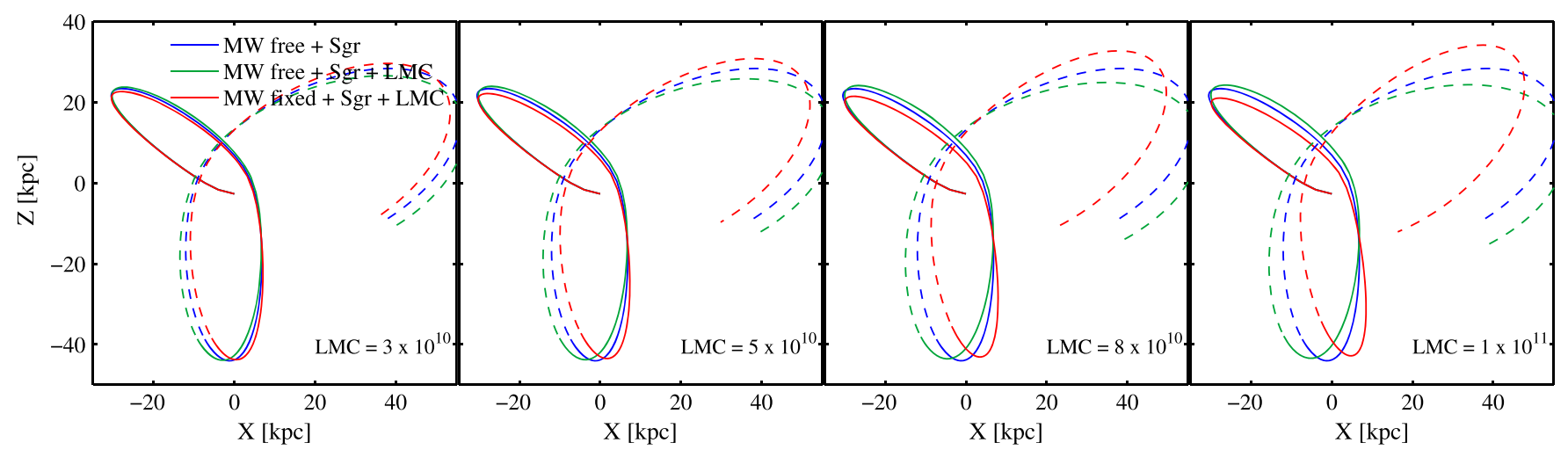

Figure 7. As in Figure 5, but for LMC models with different total masses. In each panel, the mass of the corresponding LMC model is indicated in the bottom right. In all cases, we consider a MW potential with mass $M_{\text {vir }}=1 \times 10^{12} M_{\odot}$. Note that perturbations in the Sgr-like orbits are noticeable in all simulations that include LMC models with masses $>5 \times 10^{10} M_{\odot}$. 

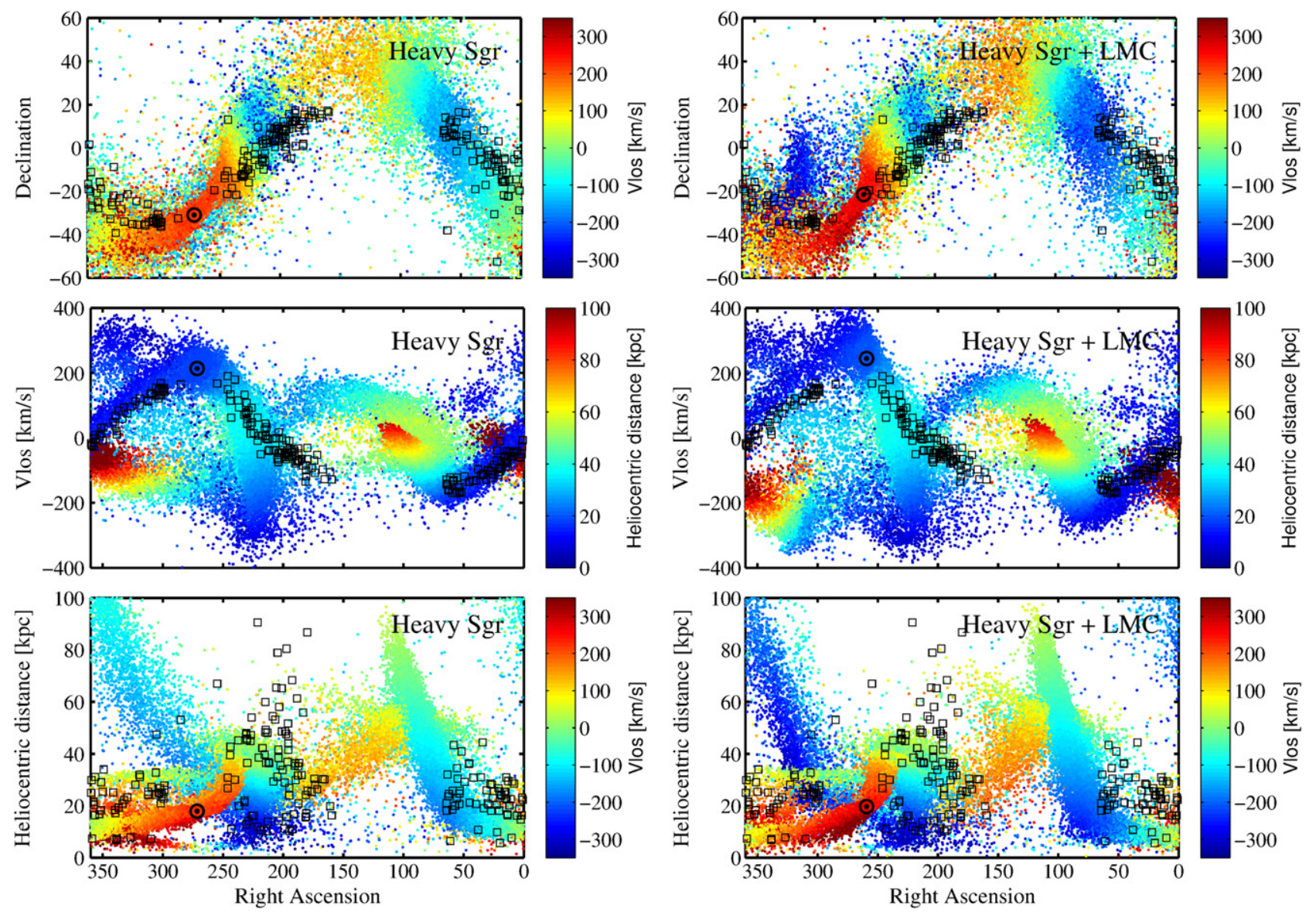

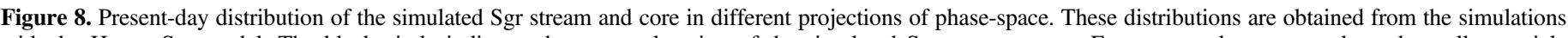

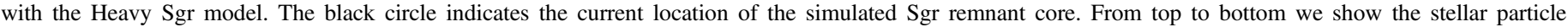

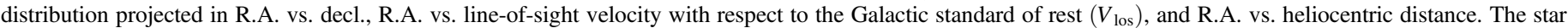

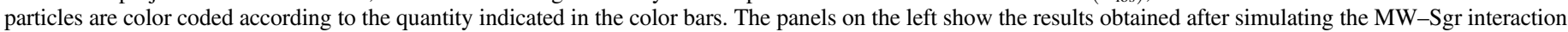

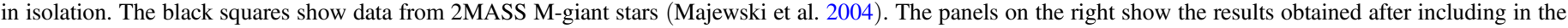

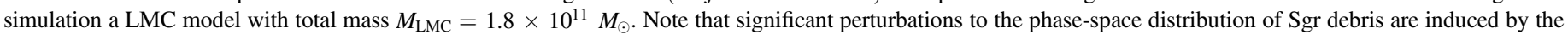
LMC. These perturbation are the result of both the torque exerted by the LMC on Sgr and the response of the MW to the LMC's gravitational pull.

Ostholt et al. 2012). As discussed in Section 3.1.2 (see also P11), the Sgr-like satellites are launched at a Galactocentric distance of $80 \mathrm{kpc}$ from the Galactic center in the plane of the MW disk, traveling vertically at $80 \mathrm{~km} \mathrm{~s}^{-1}$ toward the North Galactic Pole. To account for the mass loss that would have occurred between the crossing of the MW's virial radius and this "initial" location, the Sgr progenitor NFW mass profiles are initially truncated at the corresponding instantaneous Jacobi radius, $r_{\mathrm{J}} \approx 30$ and $23 \mathrm{kpc}$ for the Heavy and Light Sgr, respectively.

Table 3 summarizes the values of all of the parameters that describe our $N$-body models.

\subsection{An N-body Treatment of the MW+LMC+Sgr System}

The $N$-body models discussed in this section will build on the previously discussed analytic models to explore the impact of LMC perturbations in a first infall scenario on the Sgr tidal debris. To this end, we compare simulations in which the LMC is included against others in which it is not. In what follows, all simulations include fully self-consistent three-component MW models that are allowed to respond to the gravitational pull of any external source. As described in Section 5.1.2 we consider Heavy and Light Sgr models, both self-consistently initialized with spherical baryonic and DM components. The Heavy and the Light Sgr are followed for $\sim 2.1$ and $2.6 \mathrm{Gyr}$, respectively, at which time they reach a heliocentric distance of approximately $20 \mathrm{kpc}$. For more details on the setup of these simulations, we refer the reader to P11.

In the left panels of Figures 8 and 9 we show the distribution of the Sgr debris at present-day in different projections of phase-space in the Heavy and Light Sgr simulations, respectively.

In this simulation the LMC is not included, but the MW can respond to the presence of Sgr. The black squares show data from 2MASS M-giant stars (Majewski et al. 2004). As discussed by P11, although the simulated Sgr debris distributions do not precisely match all of the observed characteristics, they do produce a reasonable fit. Note that small differences in these distributions of debris and those shown in Figure S3 of P11 are probably due to the slightly different MW model used in this work.

The right panels show the same distributions, but now including in the simulations a first-infall LMC model with a total mass $M_{\mathrm{LMC}}=1.8 \times 10^{11} M_{\odot}$. The initial conditions for the LMC models were obtained by backward time integration from its present-day location in a "free" MW scenario (see Section 3) until 2.1 or 2.6 Gyrs ago, depending on the Sgr 

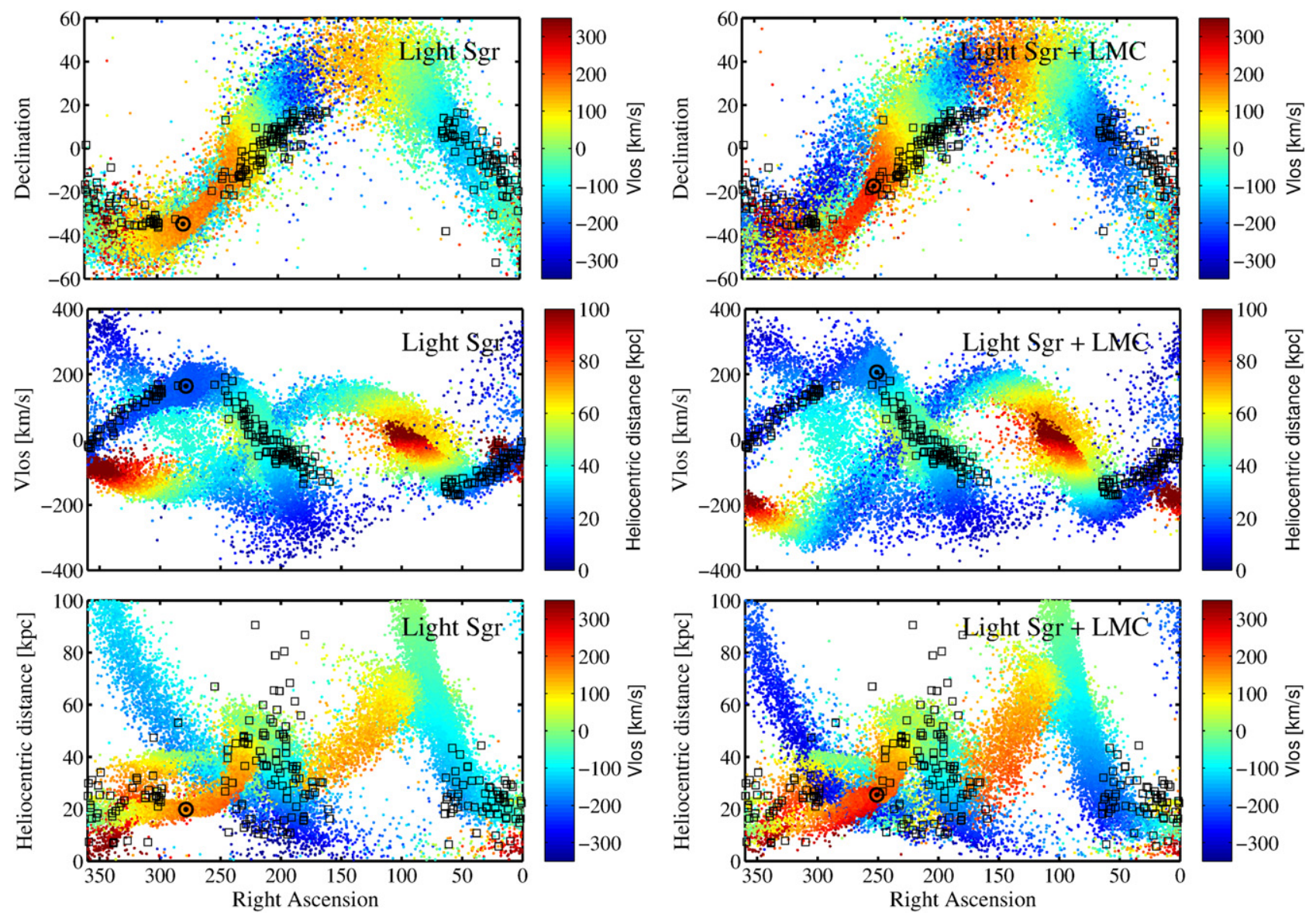

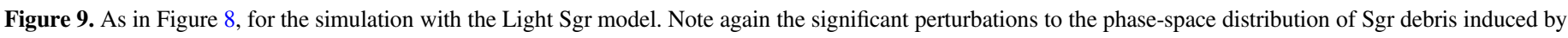
the LMC.

model. Note that due to the slight overestimation of the role of dynamical friction in the analytic calculations (see discussion in Section 3.2), the LMC ICs were iteratively calibrated by comparing the resulting LMC $N$-body orbits with their analytic counterpart. The goal of this exercise was to obtain a set of ICs for the $N$-body simulations that, at present day, yields the correct phase-space coordinates. The Sgr dwarf galaxy is launched with the same ICs as in the "LMC-less" simulations. Clearly, the addition of the LMC results in significant perturbations in the phase-space distribution of Sgr debris.

The top panels of Figures 8 and 9 show the simulated present-day Sgr stream and the remnant core projected in right ascension (R.A.) and decl. . A comparison between simulations with and without the LMC model reveals an interesting feature at R.A. $\approx 300^{\circ}$. When the LMC is accounted for, tidal debris that otherwise would overlap when projected on the sky are split into two distinguishable arms. This is true for both Sgr models, suggesting that tidal material could also be deposited in regions of the sky that are not delineated by the present-day Sgr orbital plane. Note that the two arms show both opposite heliocentric distance gradients as a function of R.A. (see bottom right panel in Figures 8 and 9) and opposite line-ofsight velocities.

The middle panels of Figures 8 and 9 show the projected Sgr distribution in R.A. versus Galactic standard of rest line of sight velocity $\left(V_{\text {los }}\right)$ space. A quick comparison between the left and right panel reveals very significant changes in the distribution of $V_{\text {los }}$. In general, adding the LMC results in a much broader distribution at all R.A.. This can be more clearly seen on Figure 10 where, as an example, we show the $V_{\text {los }}$ distributions of Heavy Sgr star particles located within $250^{\circ}<$ R. A. $<300^{\circ}$. Recall that, as shown in Figure 7, these perturbations are not just the result of the LMC torque on Sgr, but are also due to the self-consistent response of the MW to the LMC's gravitational pull. The phase-space distribution of the Sgr debris obtained when the LMC is included in the simulation results in a worse fit to the Majewski et al. (2004) data. However, in this work we have not attempted to find a set of ICs that could fit the Sgr debris in a scenario in which the LMC is included. Starting with different ICs for the Sgr orbit or a lower LMC mass could plausibly bring the velocities into better agreement. Instead, our goal is simply to explore what perturbations are induced and whether they are significant.

Perturbations to the Sgr debris phase-space distribution can also be observed in the bottom panels of Figures 8 and 9, where we show the projection onto R.A. versus heliocentric distance space. It is clear that the addition of the LMC resulted in a significant spatial redistribution of Sgr debris. Note that, independent of whether the LMC is included or not, star particles in the leading and trailing arm can reach distances of $\sim 50 \mathrm{kpc}$ (at R.A. $\approx 240^{\circ}$ ) and $\sim 100 \mathrm{kpc}$ (R.A. $\left.\approx 80^{\circ}\right),{ }^{11}$ respectively (also, see Figure S4 from P11). These different distances are similar to the leading and trailing tail's apocentric distances of the Sgr stream, as traced by B14. They find

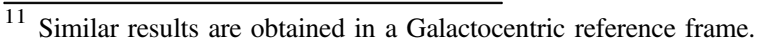




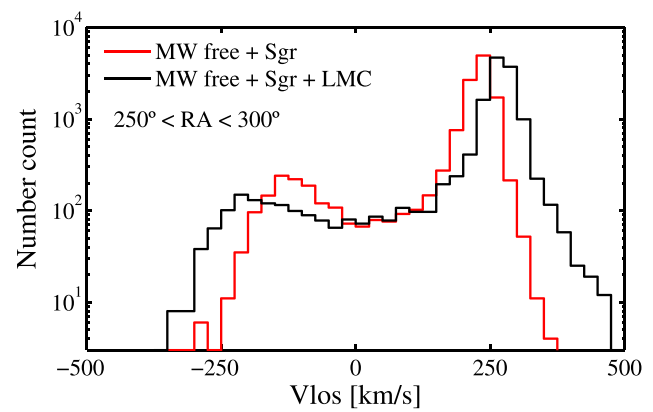

Figure 10. Distribution of lines-of-sight velocities, $V_{\mathrm{los}}$, of Heavy $\mathrm{Sgr}$ star particles located within $250^{\circ}<\mathrm{R}$. A. $<300^{\circ}$. The red line shows the results obtained in a self-consistent $N$-body simulation of the interaction between the MW and Sgr. The black line shows the results obtained when a model of the LMC is added to the simulation.

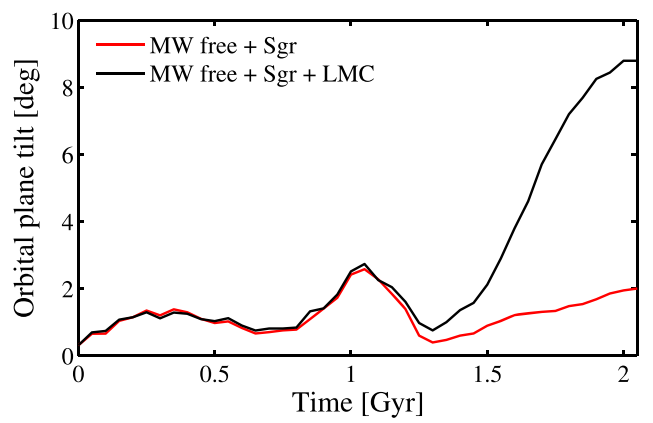

Figure 11. Time evolution of the Heavy Sgr orbital angular momentum orientation with respect to its initial direction. The red line shows the results obtained in a self-consistent $N$-body simulation of the interaction between the MW and the Sgr dwarf galaxy. The black line shows the results obtained when a model of the LMC is self-consistently added to the simulation.

$R_{\text {lead }}^{\text {apo }} \approx 48 \mathrm{kpc}$ and $R_{\text {trail }}^{\text {apo }} \approx 102 \mathrm{kpc}$, respectively. The different apocentric distances reached by the star particles in our simulations in the leading and trailing arms are merely a consequence of considering a self-gravitating Sgr model (see Choi et al. 2007; Gibbons et al. 2014).

It is also interesting to explore whether the self-consistent addition of the LMC could at least partially explain the $\approx 10^{\circ}$ difference between the mean orbital poles of the great circles associated with the debris leading and trailing Sgr (Johnston et al. 2005). In Figure 11 we show the time evolution of the Heavy Sgr orbital angular momentum orientation, $\hat{L}$. Since Sgr is launched in the $X-Z$ plane, its angular momentum initially points in the $\hat{Y}$ direction. The red line shows the angular displacement of $\hat{L}$ with respect to $\hat{Y}$ in the LMC-less scenario. As expected from a polar orbit in an axisymmetric potential, the orientation of the angular momentum remains nearly constant and close to $0^{\circ}$ at all times. The black line shows the result obtained after adding the LMC model. Clearly, as the LMC approaches the MW, the Sgr orbital plane starts to tilt with respect to its initial orientation. This tilting takes place during the last 0.5 Gyr of evolution, in good agreement with the results shown in Figure 3. At present-day, the angular displacement of $\hat{L}$ is of approximately $9^{\circ}$, similar to the value reported by Johnston et al. (2005).

\section{DISCUSSION AND CONCLUSIONS}

In this work we have performed and analyzed a set of numerical simulations using smooth and $N$-body gravitational potentials. Our goal was to explore whether the approximation of an inertial Galactocentric reference frame holds in the presence of a relatively massive LMC that is experiencing its first infall toward the MW. In a nutshell, if the LMC currently has a total mass of at least $5 \times 10^{10} M_{\odot}$, the answer is likely to be no.

To arrive at this conclusion, we have focused our efforts on two possible situations where artificially fixing the MW center of mass could have a significant effect. Our first obvious choice was to explore the implications on the orbit calculations of the LMC about the MW. Our results clearly show that the LMC's orbital period and apocentric distance are significantly shortened if we allow the MW to react to the LMC's gravitational pull. As the mass of the LMC becomes larger, and thus more comparable to the MW mass enclosed within the LMC's location, the two-body interaction becomes more relevant. Thus, the more massive the LMC, the larger the changes on its orbital periods. The change in the inferred orbital properties of our LMC-like models suggest that, even though a first-infall is still a very plausible scenario, the limiting LMC-MW mass combinations that could host a firstinfalling LMC are noticeably affected; it raises the required minimum LMC mass and disfavors MW models with $M_{\text {vir }} \geqslant 1.5 \times 10^{12} M_{\odot}$. A detailed dynamical analysis, including $N$-body models that can naturally account for the two body interaction, the LMC's tidal mass loss and dynamical friction, and a model for the time evolution of the MW potential, would be required to robustly characterize the orbital history of the different LMC mass models. This is beyond the scope of the work presented in this paper.

We have also characterized how the MW itself responds to the gravitational pull of the LMC. We find that significant changes in both the position and velocity of the MW center of mass takes place only during the last $0.3-0.5$ Gyr of evolution. It is around this time when both the MW mass enclosed within the LMC Galactocentric distance becomes comparable to the mass of the LMC itself and the distance between both galaxies becomes short enough. For example, for a LMC model with a total mass $M_{\mathrm{LMC}}=1.8 \times 10^{11} M_{\odot}$, the orbital barycenter is located at $\approx 14 \mathrm{kpc}$ from the MW center of mass at the present day. For this LMC model, the MW was displaced by $\approx 30 \mathrm{kpc}$ and its velocity changed by $\approx 75 \mathrm{~km} \mathrm{~s}^{-1}$ in this very short amount of time. Note that similar results were obtained in simulations of the collision between Andromeda and its satellite galaxy M32 (Dierickx et al. 2014).

Due to the extended nature of the MW stellar halo, not all stars are accelerated at the same rate by a massive satellite. It is thus likely that this differential acceleration will have important effects on the observable properties of extended stellar streams. For example, the distribution of Sgr debris, which covers a radial extension of $\sim 100 \mathrm{kpc}$, could be significantly perturbed due to the phase-space displacement of the Milky Way center of mass, in addition to the perturbations associated with the LMC torque (e.g., VCH13). To explore this, we integrated Sgrlike orbits in MW potentials that are allowed to freely react to the gravitational pull of the LMC. We would like to stress that in this work we have not attempted to find a set of ICs for the Sgr progenitor that could fit the Sgr debris in a scenario in which the LMC is included. The complexity behind the search for best-fitting ICs in a three-body problem scenario using fully self-consistent models is beyond the scope of this work. Instead, our goal is simply to explore whether or not such perturbations are significant. 
Our analysis showed that, indeed, the presence of the LMC introduced significant perturbations on Sgr-like orbits and their associated distribution of debris. We have confirmed previous results presented by $\mathrm{VCH} 13$, where they showed that the torque on Sgr exerted by the LMC can introduce non-negligible perturbations on its orbit and distribution of debris. However, we find that the differences between the Sgr-like orbits obtained in "free" and a "fixed" MW+LMC models are even larger that those obtained in "free" models with or without the LMC. Furthermore, we have shown that this perturbation is significant even in the scenario where the LMC is undergoing its first pericenter passage. Attempts to reproduce the Sgr stream without a model for the LMC perturbation will thus force searches for the best-fitting parameters that characterize the MW gravitational potential to artificially adjust in order to account for this perturbation.

An example is the discrepancy discussed by (Belokurov et al. 2014, B14) in the angular distance between the inferred apocenters of the Sgr leading and trailing arms. Observations suggest that this angular distance is smaller than what is predicted in a fixed logarithmic potential. Our analysis showed that the differences in the angular distances between the last two Sgr's orbital apocenters could at least be partially accounted for with both a free MW model and the inclusion of the LMC.

Another example is the $\approx 10^{\circ}$ difference between the mean orbital poles of the great circles associated with the debris leading and trailing the Sgr core, reported by Johnston et al. (2005). We find in our simulations that, due to the gravitational pull exerted by the LMC, the Sgr orbital plane tilts with respect to its initial orientation by $\approx 9^{\circ}$ during the last $0.5 \mathrm{Gyr}$ of evolution. These are just two examples of peculiar characteristics of the Sgr debris that could be naturally and, at least, partially accounted for if a fully self-consistent model of the $\mathrm{MW}+\mathrm{LMC}+\mathrm{Sgr}$ interaction is considered.

Interestingly, these results were obtained without the need for a prolate/oblate model of the Galactic DM halo. To accurately quantify the significance of these perturbations, fully self-consistent models of the $\mathrm{MW}+\mathrm{LMC}+\mathrm{Sgr}$ interactive system are required. Note that, for each combination of galactic models, a specially tailored set of initial orbital conditions for the LMC and Sgr will be required. We defer this analysis to a follow-up work.

The orbit of the LMC about the MW and the orbital history and phase-space distribution of $\mathrm{Sgr}$ debris are just two examples where perturbations induced by the MW+LMC interaction could be significant. The inferred orbital properties of other MW dwarfs, such as Carina, Fornax, Sculptor and Ursa Minor, obtained using present-day phase-space coordinates, could also be affected by such interaction if the LMC is massive enough (e.g., Angus et al. 2011; Pasetto et al. 2011). Furthermore, using HST proper-motion measurements, van der Marel et al. (2012) estimated a radial velocity of M31 with respect to the MW of $V_{\mathrm{rad}, \mathrm{M} 31}=-109.3 \pm 4.4 \mathrm{~km} \mathrm{~s}^{-1}$, and a tangential velocity $V_{\mathrm{tan}, \mathrm{M} 31}=17.0 \mathrm{~km} \mathrm{~s}^{-1}$, with $1 \sigma$ confidence region $V_{\tan , \mathrm{M} 31} \leqslant 34.3 \mathrm{~km} \mathrm{~s}^{-1}$. We have shown that if the LMC is as massive as $1.8 \times 10^{11} M_{\odot}$, the velocity of the MW center of mass could have changed by as much as $75 \mathrm{~km} \mathrm{~s}^{-1}$ in less than 0.5 Gyr. Decomposing this velocity into a tangential and radial components toward M31 yields $V_{\text {rad, } \mathrm{MW}} \approx 37 \mathrm{~km} \mathrm{~s}^{-1}$ and $V_{\mathrm{tan}, \mathrm{MW}} \approx 66 \mathrm{~km} \mathrm{~s}^{-1}$. This suggests that estimates of the Local Group mass based on timing arguments could be affected by such a two-body interaction. In addition, a significant fraction of the present-day relative velocity of M31 with respect to the Galactic center could be associated to the temporary Galactic displacement about its orbital barycenter, thus affecting the projected evolution of the MW+M31 system.

We are on the verge of the so-called Gaia era. In addition to the very accurate phase-space catalogs that we are already mining, Gaia is starting to collect phase-space information for many millions of stars. The high-quality data that will soon become available clearly calls for the development of models that are as detailed as possible, and which include all known sources of significant interactions. The results presented in this work suggest that, if the LMC is as massive as suggested by recent studies, to properly interpret this data it is essential to consider in the analyzes self-consistent MW+LMC models that are allowed to freely react to their mutual gravitational interactions.

F.A.G. and B.W.O. are supported through the NSF Office of Cyberinfrastructure by grant PHY-0941373 and by the Michigan State University Institute for Cyber-Enabled Research (iCER). B.W.O. was supported in part by NSF grant PHY 08-22648: Physics Frontiers Center/Joint Institute for Nuclear Astrophysics (JINA). D.D.C. is supported by the Universidad Nacional de La Plata, Argentina, and the Instituto de Astrofísica de La Plata, UNLP-Conicet, Argentina. G.B. acknowledges support from NASA through Hubble Fellowship grant $H S T$-HF-51284.01 A.

\section{REFERENCES}

Angus, G. W., Diaferio, A., \& Kroupa, P. 2011, MNRAS, 416, 1401 Athanassoula, E., Fady, E., Lambert, J. C., \& Bosma, A. 2000, MNRAS, 314,475

Bailin, J., \& Steinmetz, M. 2005, ApJ, 627, 647

Barden, S. C., Jones, D. J., Barnes, S. I., et al. 2010, SPIE Proc., 7735, 773509 Behroozi, P. S., Conroy, C., \& Wechsler, R. H. 2010, ApJ, 717, 379 Bekki, K. 2012, MNRAS, 422, 1957

Belokurov, V., Zucker, D. B., Evans, N. W., et al. 2006, ApJL, 642, L137 Belokurov, V., Koposov, S. E., Evans, N. W., et al. 2014, MNRAS, 437, 116 Benson, A. J., Lacey, C. G., Frenk, C. S., Baugh, C. M., \& Cole, S. 2004, MNRAS, 351, 1215

Besla, G. 2014, in Lessons from the Local Group-A Conference in Honour of David Block and Bruce Elmegreen, ed. K. C. Freeman et al. (New York: Springer), 311

Besla, G., Kallivayalil, N., Hernquist, L., et al. 2007, ApJ, 668, 949

Besla, G., Kallivayalil, N., Hernquist, L., et al. 2010, ApJL, 721, L97

Besla, G., Kallivayalil, N., Hernquist, L., et al. 2012, MNRAS, 421, 2109

Binney, J., \& Tremaine, S. 2008, Galactic Dynamics (2nd ed.; Princeton, NJ: Princeton Univ. Press)

Blumenthal, G. R., Faber, S. M., Flores, R., \& Primack, J. R. 1986, ApJ, 301, 27

Bonaca, A., Geha, M., Küpper, A. H. W., et al. 2014, ApJ, 795, 94

Bovy, J., Allende Prieto, C., Beers, T. C., et al. 2012, ApJ, 759, 131

Boylan-Kolchin, M., Bullock, J. S., Sohn, S. T., Besla, G., \& van der Marel, R. P. 2013, ApJ, 768, 140

Bryan, S. E., \& Cress, C. M. 2007, MNRAS, 380, 657

Bullock, J. S., \& Johnston, K. V. 2005, ApJ, 635, 931

Carpintero, D. D., Gómez, F. A., \& Piatti, A. E. 2013, MNRAS, 435, L63

Chandrasekhar, S. 1943, ApJ, 97, 255

Choi, J.-H., Weinberg, M. D., \& Katz, N. 2007, MNRAS, 381, 987

Conroy, C., \& Wechsler, R. H. 2009, ApJ, 696, 620

Cora, S. A., Muzzio, J. C., \& Vergne, M. M. 1997, MNRAS, 289, 253

Cui, X.-Q., Zhao, Y.-H., Chu, Y.-Q., et al. 2012, RAA, 12, 1197

Debattista, V. P., Roškar, R., Valluri, M., et al. 2013, MNRAS, 434, 2971

Deg, N., \& Widrow, L. 2014, MNRAS, 439, 2678

Dierickx, M., Blecha, L., \& Loeb, A. 2014, ApJL, 788, L38

Drake, A. J., Catelan, M., Djorgovski, S. G., et al. 2013, ApJ, 765, 154

Eyre, A., \& Binney, J. 2011, MNRAS, 413, 1852

Fox, A. J., Wakker, B. P., Barger, K. A., et al. 2014, ApJ, 787, 147

Gardiner, L. T., \& Noguchi, M. 1996, MNRAS, 278, 191 
Gibbons, S. L. J., Belokurov, V., \& Evans, N. W. 2014, MNRAS, 445, 3788 Gilmore, G., Randich, S., Asplund, M., et al. 2012, Msngr, 147, 25

Gnedin, O. Y., Brown, W. R., Geller, M. J., \& Kenyon, S. J. 2010, ApJL, 720, L108

Gnedin, O. Y., Kravtsov, A. V., Klypin, A. A., \& Nagai, D. 2004, ApJ, 616, 16

Hashimoto, Y., Funato, Y., \& Makino, J. 2003, ApJ, 582, 196

Helmi, A. 2004, ApJL, 610, L97

Hernquist, L. 1990, ApJ, 356, 359

Ibata, R. A., Wyse, R. F. G., Gilmore, G., Irwin, M. J., \& Suntzeff, N. B. 1997, AJ, 113, 634

Johnston, K. V., Law, D. R., \& Majewski, S. R. 2005, ApJ, 619, 800

Just, A., \& Peñarrubia, J. 2005, A\&A, 431, 861

Kahn, F. D., \& Woltjer, L. 1959, ApJ, 130, 705

Kallivayalil, N., van der Marel, R. P., Besla, G., Anderson, J., \& Alcock, C. 2013, ApJ, 764, 161

Keller, S. C., Schmidt, B. P., Bessell, M. S., et al. 2007, PASA, 24, 1

Koposov, S. E., Rix, H.-W., \& Hogg, D. W. 2010, ApJ, 712, 260

Koposov, S. E., Belokurov, V., Evans, N. W., et al. 2012, ApJ, 750, 80

Law, D. R., Johnston, K. V., \& Majewski, S. R. 2005, ApJ, 619, 807

Law, D. R., \& Majewski, S. R. 2010, ApJ, 714, 229

Li, Y.-S., \& White, S. D. M. 2008, MNRAS, 384, 1459

Lux, H., Read, J. I., Lake, G., \& Johnston, K. V. 2012, MNRAS, 424, L16

Majewski, S. R., Nidever, D. L., Muñoz, R. R., et al. 2009, in IAU Symp. 256, ed. J. T. van Loon, \& J. M. Oliveira (Cambridge: Cambridge Univ. Press), 51

Majewski, S. R., Skrutskie, M. F., Weinberg, M. D., \& Ostheimer, J. C. 2003, ApJ, 599, 1082

Majewski, S. R., Kunkel, W. E., Law, D. R., et al. 2004, AJ, 128, 245

McGaugh, S. S. 2012, AJ, 143, 40

McMillan, P. J. 2011, MNRAS, 414, 2446

Miyamoto, M., \& Nagai, R. 1975, PASJ, 27, 533

Mo, H. J., Mao, S., \& White, S. D. M. 1998, MNRAS, 295, 319

Moster, B. P., Naab, T., \& White, S. D. M. 2013, MNRAS, 428, 3121

Murai, T., \& Fujimoto, M. 1980, PASJ, 32, 581

Navarro, J. F., Frenk, C. S., \& White, S. D. M. 1996, ApJ, 462, 563

Newberg, H. J., Yanny, B., Grebel, E. K., et al. 2003, ApJL, 596, L191

Nidever, D. L., Majewski, S. R., \& Burton, W. B. 2008, ApJ, 679, 432
Niederste-Ostholt, M., Belokurov, V., \& Evans, N. W. 2012, MNRAS, 422, 207

Pasetto, S., Grebel, E. K., Berczik, P., Chiosi, C., \& Spurzem, R. 2011, A\&A, 525, A99

Peñarrubia, J., Belokurov, V., Evans, N. W., et al. 2010, MNRAS, 408, L26

Perryman, M., Spergel, D. N., \& Lindegren, L. 2014, ApJ, 789, 166

Perryman, M. A. C., de Boer, K. S., Gilmore, G., et al. 2001, A\&A, 369, 339

Piffl, T., Scannapieco, C., Binney, J., et al. 2014, A\&A, 562, A91

Plummer, H. C. 1911, MNRAS, 71, 460

Price-Whelan, A. M., Hogg, D. W., Johnston, K. V., \& Hendel, D. 2014, ApJ, 794, 4

Purcell, C. W., Bullock, J. S., Tollerud, E. J., Rocha, M., \& Chakrabarti, S. 2011, Natur, 477, 301

Roškar, R., Debattista, V. P., Brooks, A. M., et al. 2010, MNRAS, 408, 783

Ruhland, C., Bell, E. F., Rix, H.-W., \& Xue, X.-X. 2011, ApJ, 731, 119

Saha, A., Olszewski, E. W., Brondel, B., et al. 2010, AJ, 140, 1719

Sanders, J. L., \& Binney, J. 2013a, MNRAS, 433, 1813

Sanders, J. L., \& Binney, J. 2013b, MNRAS, 433, 1826

Shen, J., \& Sellwood, J. A. 2006, MNRAS, 370, 2

Slater, C. T., Bell, E. F., Schlafly, E. F., et al. 2013, ApJ, 762, 6

Springel, V. 2005, MNRAS, 364, 1105

Springel, V., di Matteo, T., \& Hernquist, L. 2005, MNRAS, 361, 776

Springel, V., Yoshida, N., \& White, S. D. M. 2001, NewA, 6, 79

Steinmetz, M., Zwitter, T., Siebert, A., et al. 2006, AJ, 132, 1645

van der Marel, R. P., Fardal, M., Besla, G., et al. 2012, ApJ, 753, 8

van der Marel, R. P., \& Kallivayalil, N. 2014, ApJ, 781, 121

van der Marel, R. P., Kallivayalil, N., \& Besla, G. 2009, in IAU Symp. 256, ed. J. T. van Loon, \& J. M. Oliveira (Cambridge: Cambridge Univ. Press), 81

Vera-Ciro, C., \& Helmi, A. 2013, ApJL, 773, L4

Vera-Ciro, C. A., Sales, L. V., Helmi, A., et al. 2011, MNRAS, 416, 1377

Villalobos, Á, \& Helmi, A. 2008, MNRAS, 391, 1806

White, S. D. M. 1983, ApJ, 274, 53

Yanny, B., Rockosi, C., Newberg, H. J., et al. 2009, AJ, 137, 4377

York, D. G., Adelman, J., Anderson, J. E., Jr., et al. 2000, AJ, 120, 1579

Zentner, A. R., \& Bullock, J. S. 2003, ApJ, 598, 49 